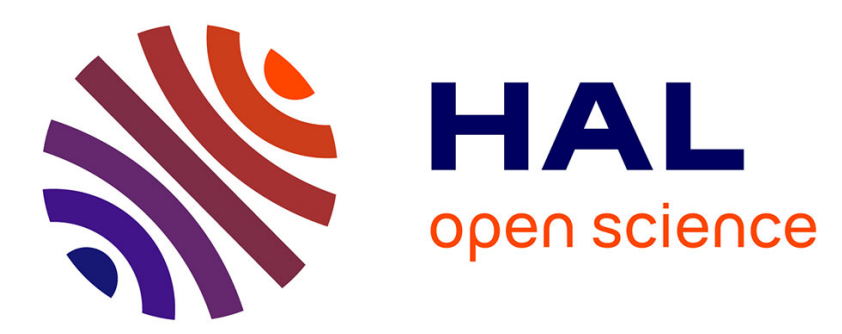

\title{
A robust-to-noise deconvolution algorithm to enhance displacement and strain maps obtained with local DIC and LSA
}

Michel Grediac, Benoît Blaysat, Frédéric Sur

\section{- To cite this version:}

Michel Grediac, Benoît Blaysat, Frédéric Sur. A robust-to-noise deconvolution algorithm to enhance displacement and strain maps obtained with local DIC and LSA. Experimental Mechanics, 2019, 59 (2), pp.219-243. 10.1007/s11340-018-00461-4 . hal-01926058

\section{HAL Id: hal-01926058 \\ https://hal.science/hal-01926058}

Submitted on 16 Dec 2018

HAL is a multi-disciplinary open access archive for the deposit and dissemination of scientific research documents, whether they are published or not. The documents may come from teaching and research institutions in France or abroad, or from public or private research centers.
L'archive ouverte pluridisciplinaire HAL, est destinée au dépôt et à la diffusion de documents scientifiques de niveau recherche, publiés ou non, émanant des établissements d'enseignement et de recherche français ou étrangers, des laboratoires publics ou privés. 


\title{
A robust-to-noise deconvolution algorithm to enhance displacement and strain maps obtained with local DIC and LSA
}

\author{
Michel Grédiac ${ }^{1 \dagger}$, Benoît Blaysat ${ }^{1}$, Frédéric Sur $^{2}$ \\ ${ }^{1}$ Université Clermont Auvergne, SIGMA, Institut Pascal, UMR CNRS 6602 \\ BP 10448, 63000 Clermont-Ferrand, France \\ ${ }^{2}$ Laboratoire Lorrain de Recherche en Informatique et ses Applications, UMR CNRS 7503 \\ Université de Lorraine, CNRS, INRIA projet Magrit, Campus Scientifique, BP 239, 54506 \\ Vandoeuvre-lès-Nancy Cedex, France \\ ${ }^{\dagger}$ corresponding author: michel.grediac@uca.fr
}

\begin{abstract}
Digital Image Correlation (DIC) and Localized Spectrum Analysis (LSA) are two techniques available to extract displacement fields from images of deformed surfaces marked with contrasted patterns. Both techniques consist in minimizing the optical residual. DIC performs this minimization iteratively in the real domain on random patterns such as speckles. LSA performs this minimization nearly straightforwardly in the Fourier domain on periodic patterns such as grids or checkerboards. The particular case of local DIC performed pixelwise is considered here. In this case and regardless of noise, local DIC and LSA both provide displacement fields equal to the actual one convolved by a kernel known a priori. The kernel corresponds indeed to the Savitzky-Golay filter in local DIC, and to the analysis window of the windowed Fourier transform used in LSA. Convolution reduces the noise level, but it also causes actual details in displacement and strain maps to be returned with a damped amplitude, thus with a systematic error. In this paper, a deconvolution method is proposed to retrieve the actual displacement and strain fields from their counterparts given by local DIC or LSA. The proposed algorithm can be considered as an extension of Van Cittert deconvolution, based on the small strain assumption. It is demonstrated that it allows enhancing fine details in displacement and strain maps, while improving the spatial resolution. Even though noise is amplified after deconvolution, the present procedure can be considered as robust to noise, in the sense that off-the-shelf deconvolution algorithms do not converge in the presence of classic levels of noise observed in strain maps. The sum of the random and systematic errors is also lower after deconvolution, which means that the proposed procedure improves the compromise between spatial resolution and measurement resolution. Numerical and real examples considering deformed speckle images (for DIC) and checkerboard images (for LSA) illustrate the efficiency of the proposed approach.
\end{abstract}

Keywords: checkerboard, digital image correlation, displacement, deconvolution, full-field measurement, grid method, localized spectrum analysis, metrology, periodic pattern, speckle, strain

This is the author-manuscript version of

M. Grédiac, B. Blaysat, F. Sur.

A robust-to-noise deconvolution algorithm to enhance displacement and strain maps obtained with local DIC and LSA.

Experimental Mechanics, SEM \& Springer, 2019.

DOI: $10.1007 / \mathrm{s} 11340-018-00461-4$ 


\section{Introduction}

Full-field measurement techniques are now widespread in the experimental mechanics community, mainly because they provide displacement and strain fields, which reveal local heterogeneities occurring when materials and structures are tested. Assessing the metrological performance of such techniques is an active research field, as illustrated by numerous recent papers on this subject, especially concerning digital image correlation (DIC). Systematic and random errors affect such measurements, and both types of errors must be characterized. Most recent papers in this field address the estimation of random error in displacement and strain maps, see [1] for instance. For a given set of images, reducing the noise level is often made by enlarging the zone over which the information is collected to obtain the displacement and strain fields at a given pixel. In classic subset-based DIC, this noise reduction can be achieved by enlarging the subset or by smoothing the displacement field obtained for a given subset size. In addition to positively impacting the noise level in displacement and strain maps, a direct but negative consequence of this type of procedure is to impair the ability of the measuring technique to distinguish small but true details in the maps since these maps become more blurred. DIC practitioners indeed know that the subset size is the main parameter, which enables them to adjust both the spatial resolution and the measurement resolution, these quantities evolving in an opposite direction when the subset size changes. In this paper, the problem raised by improving the spatial resolution (thus diminishing this quantity) without impairing the measurement resolution in the same proportion is addressed.

Classic subset-based (or local) DIC and LSA are considered here. Both techniques resolve the same problem, namely the minimization of the optical residual. The first one performs this minimization iteratively in the real domain on random patterns such as speckles, whereas the second one performs this minimization nearly straighforwardly in the Fourier domain on periodic patterns such as grids or checkerboards. We consider here the particular case of DIC performed pixelwise, in other words with a step size equal to one pixel. In this context, LSA and DIC give blurred measurement fields and this blur induces a systematic error in the measurements, which is all the higher as the frequencies involved in the true displacement and strain are high. Indeed it can be shown that displacement and strain maps are equal to their actual counterparts convolved by a kernel, which is known a priori. With DIC, this convolution kernel is given by the Savitzky-Golay filter because matching functions used to model the displacement in subsets are polynomials. This property is given and discussed in [2], which relies on [3] to establish this result. With LSA, this kernel is the window used when taking the windowed Fourier Transform of the images. This property is demonstrated in [4]. This kernel is generally a Gaussian function.

We propose here a deconvolution algorithm which enables us to eliminate, over a certain frequency range, the systematic error discussed above for DIC and LSA. The paper is organized as follows. The basics of DIC and LSA are first briefly recalled. The procedure proposed to deconvolve displacement and strain maps is then presented and discussed. The efficiency of this procedure is assessed by processing synthetic speckle and checkerboard images with DIC and LSA, respectively. Real images collected during a compression test performed on wood specimens are finally considered to illustrate the advantage of the deconvolution tool proposed here. 


\section{Digital Image Correlation and Localized Spectrum Analysis: a brief reminder}

\subsection{Problem statement}

DIC and LSA are techniques that aim at measuring the displacement fields on material surfaces. This surface is imaged before and after loading and images are then processed in order to retrieve the underlying displacement field. Both DIC and LSA rely on the principle of intensity conservation during testing. We assume here that the observed material surface is perfectly flat and that the material undergoes in-plane displacements. Moreover, the optical path and camera lens are supposed to follow the pinhole camera model, and the camera sensor is perfectly parallel with the surface under study. Consequently, the principle of intensity conservation can be written as follows at any pixel of coordinates $\underline{x}$ :

$$
f(\underline{x})=g(\underline{x}+\underline{u}(\underline{x}))
$$

where $f$ is the reference image taken before loading, and $g$ the current image. With DIC, the displacement field is estimated by minimizing the optical residual over small zones, namely the subsets. This minimization is performed with respect to the sought components of $\underline{u}$. The optical residual is, in its simplest form, the squared difference between $f(\underline{x})$ and $g(\underline{x}+\underline{u})$ integrated over the subset. Thanks to the Parseval's theorem and as demonstrated in [5] under mild assumptions, it can be shown that a unique solution of this minimization problem can be found straightforwardly in the Fourier domain if the specimen surface is marked with a periodic pattern. Consequently, DIC and LSA aim at determining an approximate value of the actual displacement $\underline{u}$ by minimizing the same function, which is the optical residual. These approximate values are denoted by $\underline{\widetilde{u}}^{\mathrm{DIC}}$ and $\underline{\widetilde{u}}^{\mathrm{LSA}}$, respectively (in the following, symbol . designates the estimate of any true quantity . measured by a given technique). Since DIC and LSA resolve the same problem, they should theoretically lead to the same solution even though they work in a different domain. Some differences in the quality of the obtained results are however observed because the underlying algorithms, which process the images, are completely different from one technique to another. The very nature of the images processed by these techniques is also different (random patterns for DIC and periodic patterns for LSA). In the next two sections, we briefly recall the main properties of DIC and LSA.

\subsection{Digital Image Correlation}

Full details on this technique can be found in [6], so we only propose here a brief reminder. Determining the displacement solution of Equation (1) is an ill-posed problem. Regularization is therefore needed, and we consider in the present study the commonly accepted classic subset-based (or local) DIC. Square subsets of dimension $h \times h$ pixels are considered. The well-known SSD ("Sum of Squared Difference") criterion is chosen here. Other criteria are presented and discussed in [7]. The main conclusion of the latter reference is that the choice of the criterion influences the robustness of the method but does not change the solution at convergence. First-degree matching functions are used here. Commercial DIC codes generally rely on such matching functions, which enable rigid body motion and homogeneous strain to be exactly described. The usual modified Gauss-Newton scheme [8] is implemented to solve

the optimization problem, with a convergence criterion defined with the value of the residual 
calculated at each iteration. Here the threshold value is set to $10^{-5}$ times the initial value of the SSD. A step equal to 1 pixel between two consecutive subsets is chosen here because assessing the ultimate metrological performance of DIC is the main objective. This choice is also made in current comparative studies carried out within the framework of the DIC challenge [9]. Consequently, a local minimization problem is written for each pixel. This increases the overall computational cost but avoids additional interpolation in order to compute the displacement field at each pixel location. Such an interpolation would inevitably negatively impact the metrological performance, and would also lead the assumptions under which the theoretical developments detailed in this paper not to be satisfied.

As discussed in [2] and regardless of noise, DIC may be considered as finding the best projection of the true displacement field $\underline{u}$ on the matching functions. Since these functions are in general polynomials, it can be shown that this projection is equivalent to a convolution of the true displacement by a suitable kernel named Savitzky-Golay filter in the literature [2, 3]. This has been recently validated with synthetic data in [5]. The approximated displacement $\underline{\widetilde{u}}^{\mathrm{DIC}}$ can therefore be considered as the convolution of the true one $\underline{u}$ with a Savitzky-Golay kernel filter $w_{\text {SG }}$. Thus

$$
\underline{\widetilde{u}}^{\mathrm{DIC}} \approx w_{\mathrm{SG}} * \underline{u},
$$

where symbol $*$ denotes bidimensional convolution.

\subsection{Localized Spectrum Analysis}

LSA processes periodic patterns. In practice this is the gray level distribution of the image of a periodic pattern, and the quantity of interest is the phase of this periodic signal [10]. In this case, the principle of intensity conservation leads to

$$
\underline{\Phi}_{f}(\underline{x})=\frac{2 \pi}{p} \underline{u}(\underline{x})+\underline{\Phi}_{g}(\underline{x}+\underline{u}(\underline{x})) .
$$

We consider here the particular case for which the periodic pattern is a checkerboard. Indeed, as discussed in a recent study [11], this pattern is optimal in terms of sensor noise propagation if it is correctly sampled in the image. Various spectral techniques such as the Geometric Phase Analysis (GPA) [12, 13], the windowed version of GPA (WGPA) [14, 15] or the Localized Spectrum Analysis (LSA) [16] are potentially available to process images of regular patterns like checkerboards in order to retrieve the phase maps of both the reference and the current images, and to deduce the resulting displacement maps. In this study, we only consider LSA. This technique relies on the windowed Fourier transform, which is also widely used in the literature for processing fringes $[17,18]$. LSA can be applied to process checkerboard patterns, as discussed in [19] and briefly recalled in Appendix 1. The main reason for which LSA is used here is that in the end, the phase returned by this method is the true one convolved by a kernel $w_{\mathrm{G}}$ known a priori and discussed hereafter. This property has been demonstrated at the first order in [4]. Thus, we can write for any image $\mathcal{I}$

$$
\underline{\widetilde{\Phi}}_{\mathcal{I}}^{\mathrm{LSA}} \approx w_{\mathrm{G}} * \underline{\Phi}_{\mathcal{I}}
$$

Basically, LSA relies on the use of the windowed Fourier transform of the signal set to the nominal frequency of the periodic pattern. Here, this frequency corresponds to $\sqrt{2} / p$, where 
$p$ is the pitch of the checkerboard. It is shown in [20] that the Gaussian window represents the best tradeoff between various constraints, so it is employed here. Note however that the theoretical developments that follow remain correct with other types of windows as long as they are continuous, symmetric, and integrate to one. The Gaussian window is defined by the following equation

$$
w_{\mathrm{G}}(\underline{x})=\frac{1}{2 \pi \sigma^{2}} e^{\frac{-\|\underline{x}\|^{2}}{2 \sigma^{2}}},
$$

where $\sigma$ denotes the standard deviation of the Gaussian. The displacement field is then deduced from the phases by using Equation 3, which leads to

$$
\underline{u}(\underline{x})=-\frac{p}{2 \pi}\left(\underline{\Phi}_{g}(\underline{x}+\underline{u}(\underline{x}))-\underline{\Phi}_{f}(\underline{x})\right)
$$

The displacement is therefore known if the phases of both the current and reference images are known. In practice however, these phases as well as the displacement are approximate values, which means that $\underline{\Phi}_{f}, \underline{\Phi}_{g}$ and $\underline{u}$ must be replaced by $\underline{\widetilde{\Phi}}_{f}, \underline{\widetilde{\Phi}}_{g}$ and $\underline{\widetilde{u}}^{\mathrm{LSA}}$, respectively. Thus

$$
\begin{aligned}
\underline{\widetilde{u}}^{\mathrm{LSA}}(\underline{x}) & =-\frac{p}{2 \pi}\left(\underline{\widetilde{\Phi}}_{g}^{\mathrm{LSA}}\left(\underline{x}+\underline{\widetilde{u}}^{\mathrm{LSA}}(\underline{x})\right)-\underline{\widetilde{\Phi}}_{f}^{\mathrm{LSA}}(\underline{x})\right) \\
& =-\frac{p}{2 \pi} \Delta \underline{\widetilde{\Phi}}^{\mathrm{LSA}}
\end{aligned}
$$

$\underline{\widetilde{u}}^{\mathrm{LSA}}$ is the solution of the above implicit equation, which is solved by using a fixedpoint algorithm discussed in [16]. Convergence is all the faster as the derivatives of the sought quantity is small. This is the case here since we deal with small strains. In practice, convergence is reached in one iteration, additional iterations bringing only negligible changes in the retrieved displacement. This is the reason why the method can be considered as "quasi-direct" and not "direct".

\subsection{Summary}

Table 1 summarizes the properties that satisfy the displacement fields returned by DIC and LSA. Both techniques rely on the same principle, namely the intensity conservation. While DIC iteratively resolves the associated minimization problem in the real domain, LSA provides a quasi-direct solution in the Fourier domain. Nevertheless, these two methods share the same important property: their outputs are equal to the convolution of the true quantity (i.e. the displacement for DIC and the phase for LSA, respectively), by a kernel which is specific to each technique. This kernel is a Savitzky-Golay filter for DIC and a Gaussian function for LSA.

The objective of the following section is to take advantage of the link existing between measured and true quantities in order to retrieve the latter from the former. This link being a convolution, we will apply a deconvolution procedure developed specifically for displacement and phase fields. This procedure is described in the following section. 


\begin{tabular}{|c|c|c|}
\hline & DIC & LSA \\
\hline $\begin{array}{c}\text { Intensity } \\
\text { conservation }\end{array}$ & $\begin{array}{l}\text { in the } \\
\text { real domain }\end{array}$ & $\begin{array}{l}\text { in the } \\
\text { Fourier domain }\end{array}$ \\
\hline Suitable for & random patterns & periodic patterns \\
\hline $\begin{array}{c}\text { Regardless of } \\
\text { noise, link } \\
\text { between true and } \\
\text { measured } \\
\text { quantities }\end{array}$ & $\underline{\tilde{u}}^{\mathrm{DIC}} \approx w_{\mathrm{SG}} * \underline{u}$ & $\begin{array}{l}\underline{\widetilde{u}}^{\mathrm{LSA}}=-\frac{p}{2 \pi} \Delta \underline{\widetilde{\Phi}}^{\mathrm{LSA}} \\
\Delta \underline{\widetilde{\Phi}}^{\mathrm{LSA}} \approx w_{\mathrm{G}} * \Delta \underline{\Phi}\end{array}$ \\
\hline $\begin{array}{l}\text { Filter involved in } \\
\text { the convolution }\end{array}$ & $\begin{array}{c}w_{\mathrm{SG}} \text { : Savitzky-Golay filter of } \\
\text { width the subset size } h \text { and } \\
\text { of order the degree of the } \\
\text { matching function }\end{array}$ & $\begin{array}{c}w_{\mathrm{G}}: \text { Gaussian window of } \\
\text { standard deviation } \sigma\end{array}$ \\
\hline
\end{tabular}

Table 1: Summary of both techniques discussed in this paper: DIC and LSA.

\section{Deconvolving the maps returned by DIC and LSA}

\subsection{Notations}

We focus here either on the displacement $\underline{\widetilde{u}}^{\text {DIC }}$ provided by DIC or on the phase $\underline{\widetilde{\Phi}}^{\text {LSA }}$ provided by LSA (and subsequently the displacement $\underline{\widetilde{u}}^{\mathrm{LSA}}$ thanks to Equation 8). In the following, both $\underline{\widetilde{u}}^{\mathrm{DIC}}$ and $\underline{\widetilde{\Phi}}^{\mathrm{LSA}}$ are denoted indifferently by $\underline{\widetilde{q}} . q$ is the corresponding true quantity, i.e. $\underline{u}$ for DIC and $\underline{\Phi}$ for LSA. The convolution kernel associated to each technique is denoted by $w$. $w$ is a Savitzky-Golay filter $w_{\mathrm{SG}}$ for DIC or a Gaussian filter $w_{\mathrm{G}}$ for LSA. According to the conclusion of the preceding section, $\underline{q}$ and $\underline{\widetilde{q}}$ are linked through the following equation

$$
\underline{\widetilde{q}}=w * \underline{q}, \text { with }\left\{\begin{array}{llll}
\underline{\widetilde{q}}=\underline{\widetilde{u}}^{\mathrm{DIC}} & , \underline{q}=\underline{u} & \text { and } w=w_{\mathrm{SG}} & \text { for DIC } \\
\underline{\widetilde{q}}=\underline{\widetilde{\Phi}}^{\mathrm{LSA}} & , \underline{q}=\underline{\Phi} & \text { and } w=w_{\mathrm{G}} & \text { for LSA }
\end{array} .\right.
$$

By definition, this convolution can also be written as follows

$$
\begin{aligned}
\underline{\widetilde{q}}\left(x_{1}, x_{2}\right) & =\iint_{\left(\eta_{1}, \eta_{2}\right) \in \Re^{2}} w\left(x_{1}-\eta_{1}, x_{2}-\eta_{2}\right) \underline{q}\left(\eta_{1}, \eta_{2}\right) \mathrm{d} \eta_{1} \mathrm{~d} \eta_{2} \\
& =\iint_{\left(\eta_{1}, \eta_{2}\right) \in \Re^{2}} w\left(\eta_{1}, \eta_{2}\right) \underline{q}\left(x_{1}-\eta_{1}, x_{2}-\eta_{2}\right) \mathrm{d} \eta_{1} \mathrm{~d} \eta_{2} .
\end{aligned}
$$

Since DIC and LSA provide quantities $\underline{\widetilde{q}}$ systematically corrupted by a convolution kernel $w$, the question is to know whether $q$ can be deduced from $\underline{\widetilde{q}}$, in other words if $\underline{\widetilde{q}}$ can be deconvolved. 
In the absence of noise, the solution of this problem is trivial since

$$
\underline{q}=\mathcal{F}^{-1}\left(\frac{\mathcal{F}(\widetilde{q})}{\mathcal{F}(w)}\right)
$$

where $\mathcal{F}$ denotes the Fourier transform and $\mathcal{F}^{-1}$ its inverse. Applying this equation to find $\underline{q}$ is called "direct deconvolution" in the following. Noise makes however deconvolution an ill-posed problem $[21,22]$. This has led to various dedicated tools, since the specificities of the problem at hand are generally taken into account in some way in its resolution. Concerning strain maps, it has been shown in [23] that off-the-shelf deconvolution tools such as the RichardsonLucy algorithm or the Wiener filter could only be applied to deconvolve displacement and strain maps if the signal-to-noise ratio was sufficiently high, which makes these tools difficult to use in real situations where displacement and strain values hardly emerge from the noise floor. The objective of the following section is to propose a robust procedure which can be applied efficiently to deconvolve $\underline{\widetilde{q}}$. The proposed algorithm is dedicated to displacement and strain map deconvolution. It goes beyond standard restoration procedures used in image processing. Its originality is to be based on the small strain assumption.

\subsection{Principle}

Contrary to the gray level distributions observed in most of real images, the quantities involved in the present problem such as the displacements and the phases generally only smoothly change throughout the surface under investigation. This enables us to consider the secondorder Taylor expansion of $\underline{q}$ to be a reliable approximation of $q$ in the neighborhood of any point of coordinates $\left(x_{1}, x_{2}\right)$. Denoting by $\underline{\eta}$ the vector of components $\eta_{1}$ and $\eta_{2}$, we have

$$
\begin{aligned}
& \underline{q}\left(x_{1}-\eta_{1}, x_{2}-\eta_{2}\right)=\underline{q}\left(x_{1}, x_{2}\right) \\
& -\sum_{i=\{u, v\}}\left(\underline{\nabla}\left(\underline{q} \cdot \underline{e}_{i}\right)\left(x_{1}, x_{2}\right) \cdot \underline{\eta}\right) \underline{e}_{i} \\
& +\frac{1}{2} \sum_{i=\{u, v\}}\left(\underline{\eta^{t}} \underline{\underline{H}}\left(\underline{q} \cdot \underline{e}_{i}\right)\left(x_{1}, x_{2}\right) \underline{\eta}\right) \underline{e}_{i}+o\left(\|\underline{\eta}\|^{2}\right),
\end{aligned}
$$

where "." denotes the scalar product between two vectors. $\left(\underline{e}_{u}, \underline{e}_{v}\right)$ is the basis where the components of $q$ are given. This basis depends on the technique (DIC or LSA) and may be different from $\left(\underline{e}_{1}, \underline{e}_{2}\right)$ (see Appendix 1 for LSA). The components of $\underline{q}$ in the $\left(\underline{e}_{u}, \underline{e}_{v}\right)$ basis are denoted by $q_{u}$ and $q_{v}$. The derivation operators used here are defined as follows

$$
\left\{\begin{array}{l}
\underline{\nabla}(y) \text { is the gradient of } y, \text { i.e. }[\underline{\nabla}(y)]_{i}=\frac{\partial y}{\partial x_{i}}=y_{, i}, \text { with } i \in\{1,2\} \\
\underline{\underline{H}}(y) \text { is the Hessian matrix of } y \text {, i.e. }[\underline{\underline{H}}(y)]_{i j}=\frac{\partial^{2} y}{\partial x_{i} \partial x_{j}}=y_{, i j}, \text { with }(i, j) \in\{1,2\}^{2}
\end{array},\right.
$$


where $y$ is twice continuously differentiable. Thanks to this Taylor expansion, Equation 11 can be approximated and $\underline{\widetilde{q}}$ can be written as follows:

$$
\begin{aligned}
& \underline{\widetilde{q}}\left(x_{1}, x_{2}\right) \approx \underline{q}\left(x_{1}, x_{2}\right) \iint_{\left(\eta_{1}, \eta_{2}\right) \in \Re^{2}} w\left(\eta_{1}, \eta_{2}\right) \mathrm{d} \eta_{1} \mathrm{~d} \eta_{2} \\
& -\sum_{i=\{u, v\}}\left(\underline{\nabla}\left(q_{i}\right) \cdot \iint_{\left(\eta_{1}, \eta_{2}\right) \in \Re^{2}} w\left(\eta_{1}, \eta_{2}\right) \underline{\eta} \mathrm{d} \eta_{1} \mathrm{~d} \eta_{2}\right) \underline{e}_{i} \\
& \quad+\frac{1}{2} \sum_{i=\{u, v\}}\left(\sum_{k, l=1}^{2} q_{i, k l} \iint_{\left(\eta_{1}, \eta_{2}\right) \in \Re^{2}} w\left(\eta_{1}, \eta_{2}\right) \eta_{k} \eta_{l} \mathrm{~d} \eta_{1} \mathrm{~d} \eta_{2}\right) \underline{e}_{i} .
\end{aligned}
$$

The above equation simplifies because $w$ integrates to one and $\iint w\left(\eta_{1}, \eta_{2}\right) \underline{\eta} \mathrm{d} \eta_{1} \mathrm{~d} \eta_{2}=\underline{0}$ for reasons of symmetry. Hence $\underline{\widetilde{q}}$ satisfies

$$
\underline{\widetilde{q}}\left(x_{1}, x_{2}\right) \approx \underline{q}\left(x_{1}, x_{2}\right)+\underline{\delta q}\left(x_{1}, x_{2}\right),
$$

where $\underline{\delta q}$ can be seen as a correction term defined by

$$
\underline{\delta q}\left(x_{1}, x_{2}\right)=\frac{1}{2} \sum_{i=\{u, v\}} \sum_{k, l=1}^{2} q_{i, k l} I_{k l} \underline{e}_{i} .
$$

$I_{k l}$ is defined by the following second-order moment

$$
I_{k l}=\iint_{\left(\eta_{1}, \eta_{2}\right) \in \Re^{2}} w\left(\eta_{1}, \eta_{2}\right) \eta_{k} \eta_{l} \mathrm{~d} \eta_{1} \mathrm{~d} \eta_{2}
$$

For DIC, $w$ is a Savitzky-Golay kernel. It is discrete by definition. We stick here with a continuous formulation of the problem for the sake of generality, but it can easily be adapted to the discrete case. $\left(I_{k l}\right)_{(k, l)=\{1,2\}^{2}}$ must be integrated numerically for DIC. For LSA, $\left(I_{k l}\right)_{(k, l)=\{1,2\}^{2}}$ relies on closed-form expressions which can be integrated analytically. This gives $I_{11}=I_{22}=\sigma^{2}$ and $I_{12}=I_{21}=0$ if a Gaussian window is used, which is the case here. Thus we have

$$
\underline{\delta \Phi}^{\mathrm{LSA}}\left(x_{1}, x_{2}\right)=\frac{\sigma^{2}}{2}\left[\sum_{i=\{u, v\}}\left(\Phi_{i, 11}+\Phi_{i, 22}\right) \underline{e}_{i}\right] .
$$

In both cases, the calculation of the correction term $\delta q$ involves the true quantity $q$, which is unknown. In practice, this issue is tackled by using a fixed-point algorithm. Starting by replacing the true quantity $\underline{q}$ by the measured quantity $\underline{\widetilde{q}}$, the correction terms is iteratively updated by replacing, in its expression defined in Equation 17, the true value $\underline{q}$ by its estimate found at the preceding iteration. Thus

$$
\left\{\begin{aligned}
& \underline{\widetilde{q}}^{0}=\underline{\widetilde{q}} \\
& \underline{\underline{q}}^{\mathrm{it}+1}=\underline{\widetilde{q}}-\underline{\delta \widetilde{q}}^{\mathrm{it}} \\
& \text { with } \underline{\delta \widetilde{q}^{\mathrm{it}}}=\frac{1}{2} \sum_{i=\{u, v\}} \sum_{k, l=1}^{2} \widetilde{q}_{i, k l}^{\mathrm{it}} I_{k l} \underline{e}_{i} .
\end{aligned}\right.
$$


This procedure can be seen as an iterative deconvolution procedure suitable for displacements obtained with DIC and for phases obtained with LSA.

\subsection{Remarks}

Several remarks can be drawn at this stage:

- This procedure is a post-processing step of local DIC (applied pixelwise) and LSA. It can therefore be used separately from DIC and LSA themselves. The sole ingredients to be known before starting this post-processing procedure are the size $h$ of the subset and the degree of the matching functions for DIC, and the standard deviation $\sigma$ of the Gaussian kernel for LSA.

- No proof of convergence of this fixed-point scheme is proposed here. Its convergence will thus be studied with numerical simulations in the following section.

- This procedure is proposed under some assumptions. In particular, we perform in Equation 13 a Taylor expansion of $\underline{q}\left(x_{1}-\eta_{1}, x_{2}-\eta_{2}\right)$ in the neighborhood of $\left(x_{1}, x_{2}\right)$. This approximation is all the more valid as $\eta_{1}$ and $\eta_{2}$, which are dummy coordinates involved in the calculation of the corrective term in Equation 17, are small quantities. The magnitude of $\eta_{1}$ and $\eta_{2}$ in the calculation of the correction term is governed by the size of the domain of integration, thus by the subset size $h$ for DIC and by the size of the Gaussian window for LSA. The latter depends on the standard deviation $\sigma$ of the Gaussian function. Hence we can expect that the greater the value of $h$ and $\sigma$, the less accurate the procedure. The simulations discussed below show that reality is however more subtle.

- Orders strictly higher than 2 can be considered in the Taylor expansion. Odd orders lead however to moments of the analysis window which are null for reasons of symmetry. Higher even order terms can also be considered, but it has been checked through numerical simulations (not shown here) that the influence of additional terms such as the fourth-order ones is negligible.

- Interestingly, we can predict that a second-degree matching function instead of a firstdegree one leads to a null corrective term, so the deconvolution procedure as defined here does not work in this case. Indeed, by definition, a Savitzky-Golay filter of degree $d$ returns the value of a polynomial interpolation of degree $d$ of the underlying signal at the center of the window. Since quadratic functions are perfectly fitted as soon as $d>1$, and since second-order moments correspond to the value of the SG filter at the origin of a quadratic function equal to 0 at this point, these moments are null in this case, and so the correction term. Additional terms in the Taylor development should be accounted for to see if deconvolution could be applied with second-degree matching function, but this issue is out of the scope of the present paper.

- The second derivatives involved in Equation 20 are performed here by convolution. The derivative kernel is a "Mexican hat" function, i.e. the second derivative of a Gaussian function. Its standard deviation is denoted by $\sigma_{\mathrm{H}}$ in the following. 
- $\underline{q}$ is obtained with experimental data which are corrupted by noise, and so the displacement and strain components. Hence it is necessary to test the robustness of the procedure described in Equation 20 to see to which extent the benefit brought about by deconvolution in terms of spatial resolution is counterbalanced by a higher noise in the deconvolved maps.

As a more general remark and concerning the originality of the proposed deconvolution algorithm compared to other algorithms already available in the literature, it can be said that the procedure proposed here can be seen as an extension of Van Cittert deconvolution [24]. When seeking for $\underline{q}$ linked to $\underline{q}$ by $\underline{\widetilde{q}}=w * \underline{q}$, the Van Cittert iterative algorithm is defined by:

$$
\left\{\begin{array}{l}
\widetilde{\widetilde{q}}^{0}=\widetilde{\widetilde{q}} \\
\underline{\widetilde{q}}^{\mathrm{it}+1}=\underline{\widetilde{q}}-\left(w * \underline{\tilde{q}}^{\mathrm{it}}-\underline{\widetilde{q}}^{\mathrm{it}}\right) .
\end{array}\right.
$$

Indeed, if the series $\underline{\underline{q}}^{\text {it }}$ converges to $\underline{q}^{\infty}$, the limit $\underline{q}^{\infty}$ is a solution of $\underline{\widetilde{q}}=w * \underline{q}^{\infty}$.

It is important to note that the Van Cittert algorithm does not always converge. Indeed the Fourier transform being linear, we can write:

$$
\mathcal{F}\left(\underline{\widetilde{q}}^{\mathrm{it}+1}\right)=\mathcal{F}(\underline{\widetilde{q}})+\left(\mathcal{F}\left(\underline{\widetilde{q}}^{\mathrm{it}}\right)-\mathcal{F}(w) \mathcal{F}\left(\underline{\widetilde{q}}^{\mathrm{it}}\right)\right) .
$$

Thus,

$$
\mathcal{F}\left(\underline{\widetilde{q}}^{\mathrm{it}}\right)=\sum_{k=0}^{\text {it }}(1-\mathcal{F}(w))^{k} \mathcal{F}(\underline{\widetilde{q}})
$$

for any it $>0$.

This geometric series converges for a given frequency $\xi$ if and only if $|1-\mathcal{F}(w)(\xi)|<1$. In this case, since $\sum_{k=0}^{+\infty}(1-\mathcal{F}(w)(\xi))^{k}=1 / \mathcal{F}(w)(\xi), \mathcal{F}\left(\underline{q}^{i t}\right)(\xi)$ converges to $\mathcal{F}(\widetilde{q})(\xi) / \mathcal{F}(w)(\xi)$, which corresponds to the direct deconvolution defined by Equation 12. The Fourier transform of any symmetric window $w$ being a real function, this means that if the transfer function $\mathcal{F}(w)$ takes non-positive values or values larger than 2, then the Van Cittert algorithm does not give a correct solution. In some situations, deconvolution is performed on frequency ranges for which the transfer function satisfies the above requirement which ensures convergence. This leads to the so-called soft deconvolution in the literature on fluid mechanics [25]. We shall come back on this in Section 5.2.

Since the Van Cittert algorithm is equivalent to direct deconvolution when it converges, it is not robust to noise. In practice it must be stopped after a few iterations. In contrast to this approach, the proposed algorithm consists in replacing $w * \widetilde{q}^{\text {it }}$ by its second-order Taylor expansion, which means that only second derivatives of $\underline{q}^{\text {it }}$ are involved in $w * \underline{q}^{\text {it }}-\underline{q}^{\text {it }}$. These derivatives being numerically estimated through a convolution with a Mexican hat function derived from a Gaussian function of standard deviation $\sigma_{\mathrm{H}}$, they are robust to noise if $\sigma_{H}$ is large enough with respect to noise amplitude. In this study, $\sigma_{H}=2.1$ pixels has been observed to be the optimal value. We can therefore conclude that the proposed iterative algorithm is a robust-to-noise extension of Van Cittert deconvolution, based on the small strain assumption.

Let us now check the efficiency of the proposed deconvolution algorithm. We present first the synthetic data used for these simulations. The results obtained with these data are then presented and discussed in turn for DIC and LSA. 


\section{Definition of the synthetic data and assessment of the per- formance}

\subsection{Definition of the synthetic displacement field}

The synthetic displacement field used here has already been introduced in [23], and then used in $[16,19]$ to test and compare the metrological performance of various full-fields measuring techniques. It is also currently used by the SEM DIC challenge to generate reference displacement fields, which are used for testing various DIC packages [9]. The horizontal displacement is null, i.e. $\underline{u}=u_{2} \underline{e}_{2}$. A simple harmonic function is chosen for the vertical displacement (see Figure 1). It is such that the mid-height is a horizontal axis of symmetry along which the displacement is constant. Thus

$$
u_{2}\left(x_{1}, x_{2}\right)=0.5 \cos \left(\frac{2 \pi}{p_{\text {wave }}\left(x_{1}\right)}\left(x_{2}-H / 2\right)\right),
$$

where $H$ is the height of the image. The period $p_{\text {wave }}$ is such that it gently and linearly changes along direction 1 :

$$
p_{\text {wave }}=p_{\text {wave }}^{\text {mini }}+\frac{p_{\text {wave }}^{\text {maxi }}-p_{\text {wave }}^{\text {mini }}}{L} x_{1}
$$

where $L$ is the length of the image. $p_{\text {wave }}^{\text {mini }}$ and $p_{\text {wave }}^{\text {maxi }}$ are the minimum and maximum values of the period of the sinusoidal displacement, respectively. The different parameters chosen here are $L=2000$ pixels, $H=501$ pixels, $p_{\text {wave }}^{\text {mini }}=10$ pixels and $p_{\text {wave }}^{\text {maxi }}=150$ pixels.

The amplitude of the vertical displacement is equal to 0.5 pixel in order to minimize the influence of image interpolation for DIC when it reaches its minimum and maximum values, in particular along the horizontal axis of symmetry $\Delta$ defined above. Indeed it is worth remembering that DIC relies on image interpolation. This introduces an extra bias in the displacement, which is called interpolation error in the DIC literature. This bias is however null in the case of integer displacement or, for reasons of symmetry, for a displacement equal to 0.5 pixel. This justifies our choice of an amplitude of the displacement equal to 0.5 pixel. The frequency of the wave decreases from the left to the right. Hence considering in the following the cross-section of the maps $\underline{\widetilde{q}}$ for different techniques along the horizontal axis of symmetry provides valuable information on the loss of amplitude as a function of the frequency of the displacement, and on its enhancement obtained by employing the proposed deconvolution procedure. 


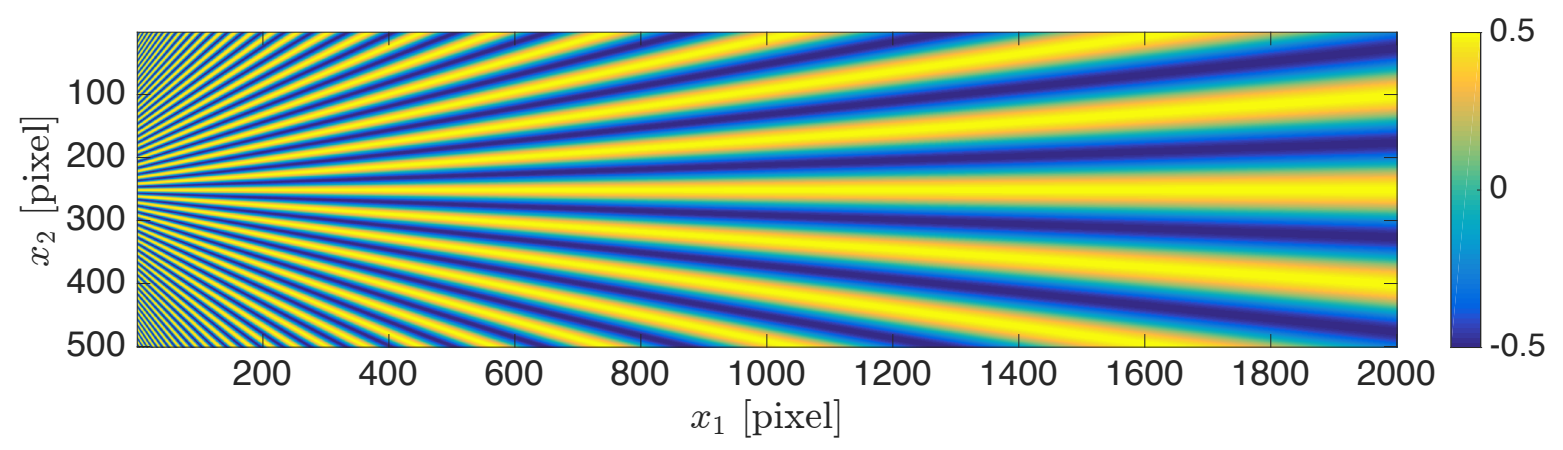

Fig. 1: Vertical reference displacement $u_{2}$

\subsection{Image generation}

The synthetic displacement $\underline{u}$ defined above was used to generate several pairs of synthetic images.

Concerning DIC, the images of the DIC challenge considered here (one reference and one deformed synthetic speckles) were generated by using the speckle pattern generator proposed in [26]. Its main feature is that no interpolation is performed while rendering the deformed speckle patterns. This is a positive point because interpolation potentially induces errors. The parameters driving the speckle pattern generator program are chosen to mimic at best a real speckle (see $[26,9]$ for more details). A closeup view of one of these speckle patterns is shown in Figure 2-a.

Concerning LSA, synthetic checkerboard images are defined as a product of two sine functions of pitch $p$, whose phases are modulated by using the same displacement as that used for DIC (see Equations 24). A Gauss integration scheme was implemented in order to mimic intensity integration. As for the speckles, no interpolation is required, the values at integer coordinates in the deformed coordinate system being obtained by applying Equations 24 at points of non-integer coordinates, which are the preimages of the integer coordinate points under $\underline{u}$. The coordinates of these preimages are obtained with a Newton-Raphson algorithm. Finally, the pitch of the checkerboard is set to $p=6$ pixels in the synthetic checkerboard images. Each feature of the pattern (here each square of the checkerboard) is therefore encoded with 3 pixels, which is the quantity suggested in [27] to fulfill the Nyquist-Shannon good sampling condition for speckles. A closeup view of a typical synthetic checkerboard is shown in Figure 2-b.

Finally, noise was added to all these synthetic images, which were registered as 8-bit images. In real cameras, sensor noise is heteroscedastic, which means that its standard deviation $\sigma_{\text {image }}(x, y)$ depends on the gray level $s(x, y)$. A popular model is to make the variance linearly dependent on the gray level, the standard deviation being written at any pixel as follows:

$$
\sigma_{\text {image }}(x, y)=\sqrt{a s\left(x_{1}, x_{2}\right)+b},
$$

where $a$ and $b$ are parameters which depend of the camera. In the DIC-challenge images used here for DIC, $a=0.0342, b=0.2679$, so these values were also used to add noise in the checkerboard images. 


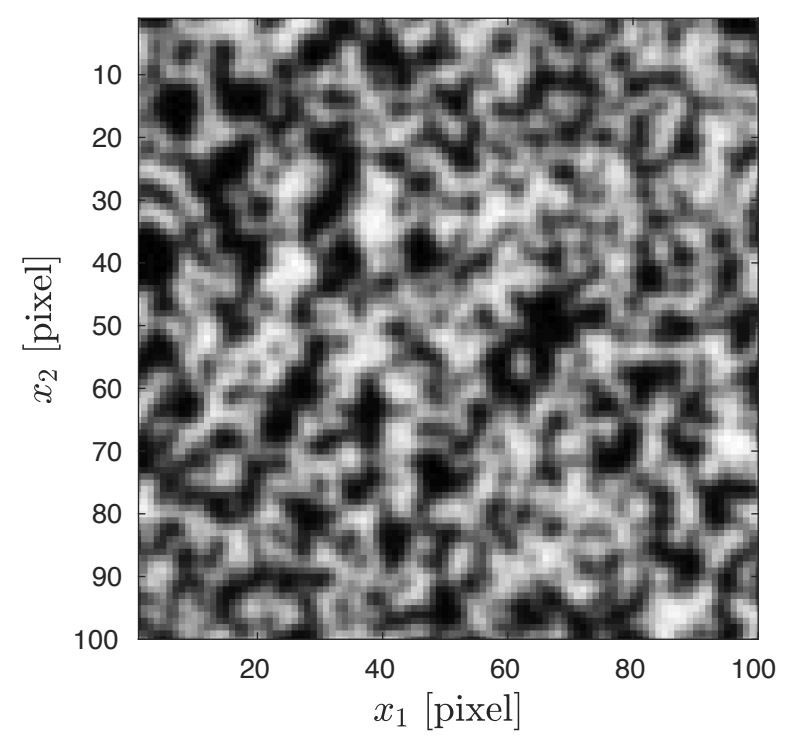

(a) Speckle reference image

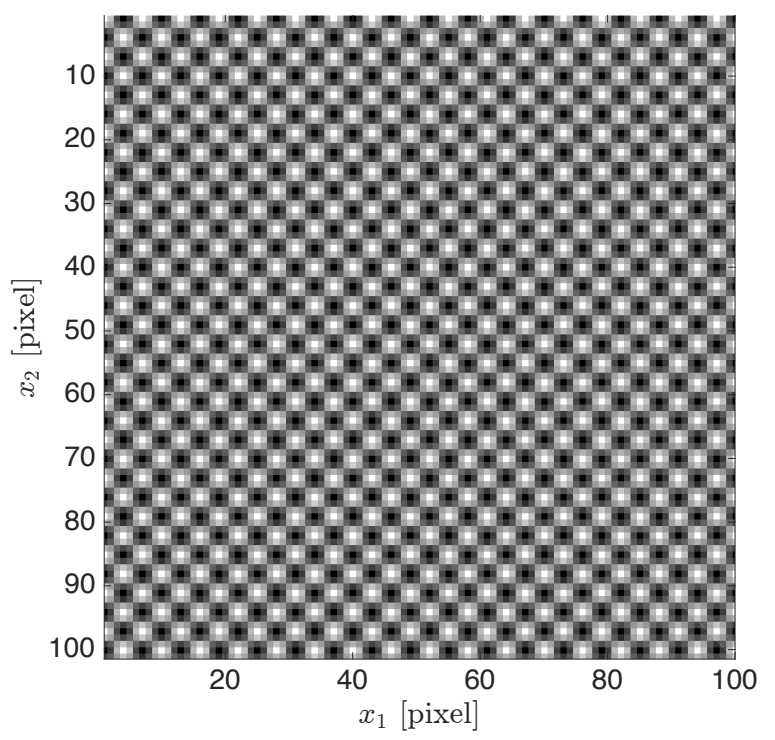

(b) Checkerboard reference image

Fig. 2: Closeup view of the artificial reference speckle image (a) and the reference checkerboard image (b) used in this study. The artificial speckle image is obtained by using the procedure described in [26].

\subsection{Estimation of the metrological performance}

Several parameters enable us to characterize the metrological performance of the two full-field measurements studied here. The parameters considered here are the bias, the displacement resolution, the spatial resolution and the metrological performance efficiency indicator. The latter is obtained by multiplying the displacement resolution by the spatial resolution, so it enables us to assess if the improvement (thus a decrease) of the spatial resolution during the deconvolution procedure is counterbalanced by an impairment (thus an increase) of the displacement resolution. The definition if these parameters and the corresponding notations is given in Appendix 2. The corresponding notations are recalled in Table 2. The procedure used to estimate the first three parameters is explained in the following section.

\section{Minimization of the optical residual in the real domain: speckle and DIC}

\subsection{Improvement of the tradeoff between spatial resolution and measure- ment resolution}

Classic subset-based DIC is applied on the pair of reference and deformed speckle patterns. We choose a subset size $h=21$ pixels for this first calculation. This choice is arbitrary but the influence of the size of the subset is discussed below. Figure 3 shows the cross-section along the horizontal axis of symmetry $\Delta$ of the displacement field retrieved after different 


\begin{tabular}{|c|c|c|c|}
\hline Parameter & Definition & Notation & SI \\
\hline $\begin{array}{c}\text { Measurement } \\
\text { resolution }\end{array}$ & $\begin{array}{c}\text { Standard deviation of the } \\
\text { noise-induced displacement } \\
\text { fluctuations }\end{array}$ & $\sigma_{u}$ & {$[$ pixel] } \\
\hline Bias & Relative loss of amplitude & $\lambda(\%)$ & {$[-]$} \\
\hline $\begin{array}{c}\text { Spatial resolution } \\
\text { Cutoff displacement wave } \\
\text { length for a given bias } \lambda\end{array}$ & $\ell_{\lambda}$ & {$[$ pixel] } \\
\hline $\begin{array}{c}\text { Metrological } \\
\text { performance efficiency } \\
\text { indicator }\end{array}$ & $\begin{array}{c}\text { Product between the } \\
\text { measurement resolution and } \\
\text { the spatial resolution }\end{array}$ & $\alpha_{\lambda}$ & {$[\text { pixel }]^{2}$} \\
\hline
\end{tabular}

Table 2: Parameters and notations used to characterize the metrological performance. More details are given in Appendix 2

iterations of the deconvolution procedure applied on the displacement field returned by DIC. The period of the sine wave $p_{\text {wave }}$ used to model the displacement is directly reported on the horizontal axis, this quantity being then useful to determine the spatial resolution (the affine link between $x_{1}$ and $p_{\text {wave }}$ is given in Equation 25). Bold lines represent the curves obtained for the first and last (hence the twentieth) iterations.

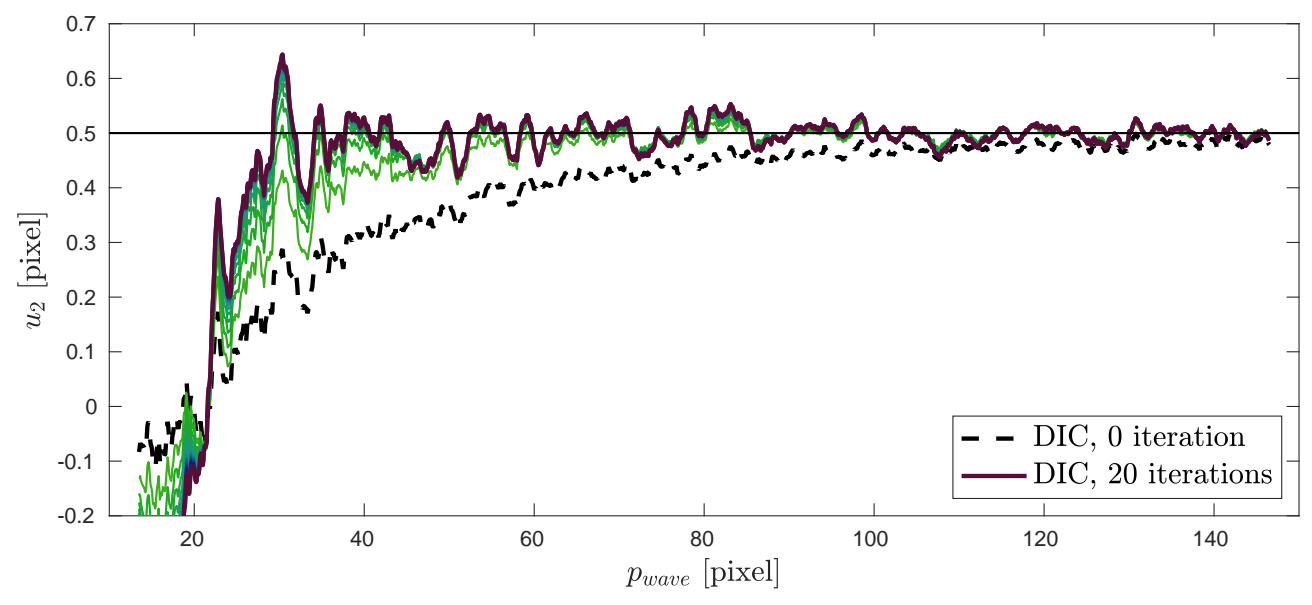

Fig. 3: Displacement obtained with speckle and DIC. Cross-section along $\Delta$ of the displacement map returned by the deconvolution procedure during the first 20 iterations. The color of the lines turns from green to mauve as the iteration number increases.

Several remarks can be drawn from the curves depicted in this figure. 
- The most striking point is that the positive effect of the deconvolution procedure is clearly visible. Indeed the curves rapidly tend to the reference value $\left(u_{2}=0.5\right.$ pixels) up to a certain value of the period $p_{\text {wave }}$ equal to the inverse of the cutoff frequency characterizing the procedure. During the iterative procedure, this is reflected by a decrease (thus an improvement) of the spatial resolution $\ell_{\lambda}^{\mathrm{DIC}}$ as defined in Appendix 2. This value is found here by calculating numerically the period $p_{\text {wave }}$ for which the amplitude of the displacement returned after deconvolution is equal to $0.5(1-\lambda)$. The displacement fields being noisy, the curves are locally smoothed to find this value. A mere sliding averaging window of width 200 pixels is applied for this purpose. "Nonnoisy" images can also directly be used but similar results are obtained.

- The curves of the successive iterations progressively draw near and overlay each other after about 6 iterations, which illustrates that the procedure converges here. In the following calculations, we will therefore systematically perform 10 iterations at most. A lower number of iterations is needed to converge with subset sizes smaller than $h=$ 21 pixels, so choosing 10 iterations is conservative.
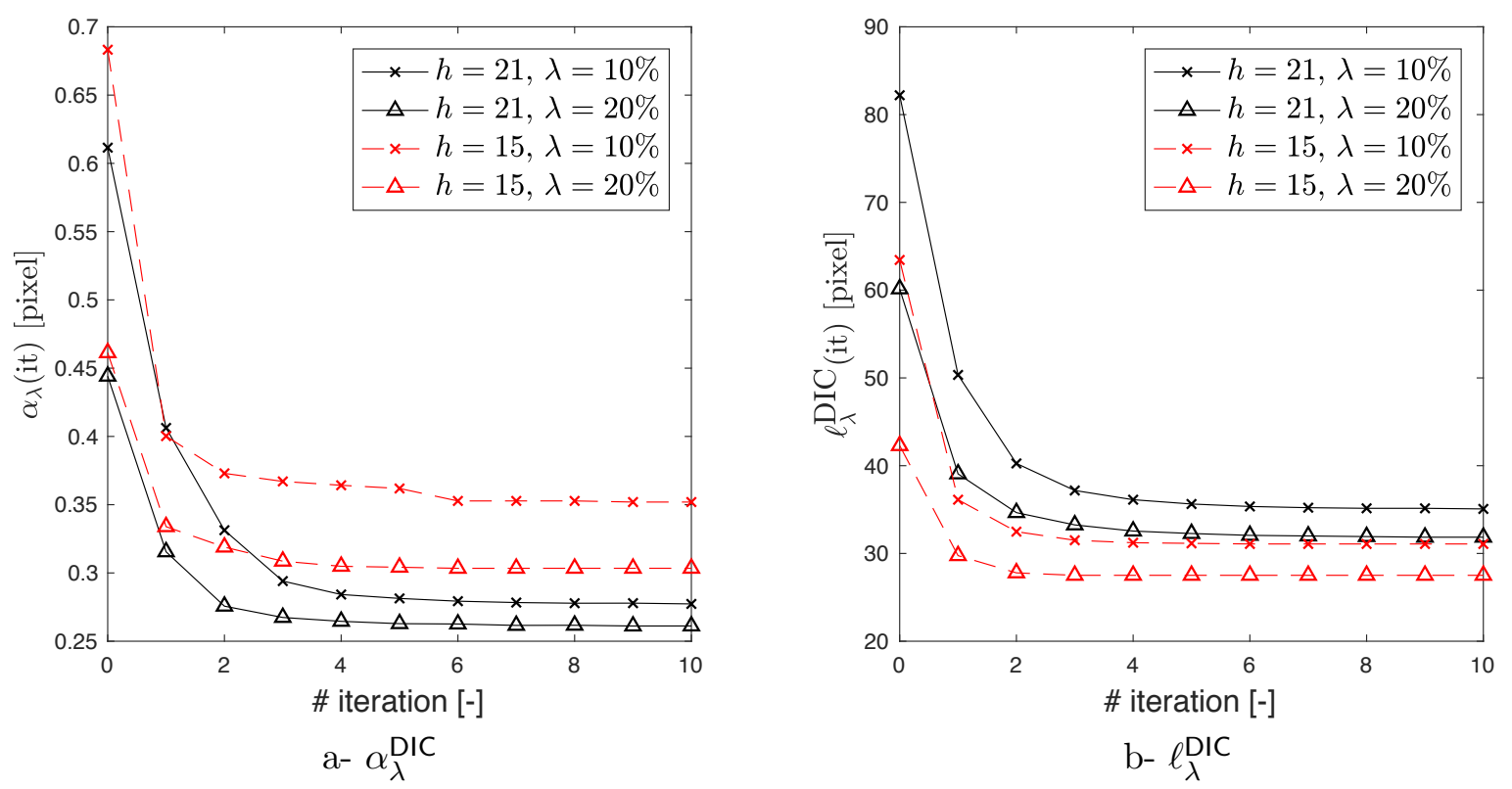

Fig. 4: $\alpha_{\lambda}^{\mathrm{DIC}}$ and $\ell_{\lambda}^{\mathrm{DIC}}$ during the first 10 iterations for $h \in\{15,21\}$ and $\lambda \in\{10 \%, 20 \%\}$.

- The images processed here are deliberately noisy and this noise propagates up to the displacement field. An important point, which can be seen to the naked eye, is that the shape of the features observed along the curves are reproduced throughout the deconvolution procedure, and with an increasing amplitude. The noise level is quantified by the displacement resolution as defined in Appendix 2. This quantity is determined here by subtracting a first displacement map obtained with a pair of noisy images, and a second one obtained with "non-noisy" images. The equivalent standard deviation $\sigma_{u}^{\text {DIC }}$ over the displacement map is then calculated and considered to be equal to the displacement resolution. The question is now to know if the total error (the sum of the 
systematic and random errors) is not greater after deconvolution. It can be seen that the curve after 10 iterations is globally closer to the reference one than the curve obtained with classic DIC (named "iteration 0" in the figure), which illustrates the fact that the total error is smaller after deconvolution. The root-mean-square error could quantify this difference, but for the sake of consistency with other recent studies $[16,5]$, we prefer here to settle this question by considering the metrological efficiency parameter $\alpha_{\lambda}^{\text {DIC }}$ (see Table 2), and estimating its change throughout the iterative procedure. Figure 4-a represents $\alpha_{10}^{\mathrm{DIC}}$ and $\alpha_{20}^{\mathrm{DIC}}$ for two different values of the subset size, namely $h=15$ and $h=21$ pixels. Noise is filtered by applying a low-pass filter in the Fourier domain, as justified and explained in Section 5.2. The main conclusion which can be drawn from these curves is that the value of $\alpha_{\lambda}^{\mathrm{DIC}}$ regularly decreases and rapidly stabilizes. This proves that the deconvolution procedure really improves the metrological performance of DIC in terms of tradeoff between spatial resolution and measurement resolution. $\lambda$ is fixed arbitrarily in turn to $10 \%$ and $20 \%$ but it can be seen that the same conclusion can be drawn for both values of $\lambda$.

- Two other interesting points can be noted in Figure 4-a. First it can be seen that for classic DIC ("iteration=0") and for a given value of $\lambda$, the values of $\alpha_{\lambda}^{\mathrm{DIC}}$ obtained for two different values of the subset size are nearly the same, which confirms that $\alpha_{\lambda}^{\mathrm{DIC}}$ can be considered as an intrinsic parameter characterizing a given technique, as rigorously demonstrated in the particular case of LSA $[16,28]$. The second point is that for a given value of $\lambda, \alpha_{\lambda}^{\mathrm{DIC}}$ obtained with the greatest subset size of the two cases studied here ( $h=21$ pixels), is significantly lower (thus better) than $\alpha_{\lambda}^{\mathrm{DIC}}$ obtained with the smallest subset size ( $h=15$ pixels). The reason is given in Figure 4-b, where the spatial resolution $\ell_{\lambda}^{\mathrm{DIC}}$ is plotted throughout the iterative procedure. As expected, the spatial resolution for classic DIC ("iteration=0") is smaller for the smallest subset size $h$. However, the value of $\ell_{\lambda}^{\mathrm{DIC}}$ in the case $h=21$ pixels becomes close to that obtained in the case $h=15$ pixels after deconvolution. Since at the same time, the noise level is always lower for the greatest window (this result is quite obvious, so it is not illustrated here), the product between the two quantities, which defines parameter $\alpha_{\lambda}^{\mathrm{DIC}}$, is lower for $h=21$ pixels than for $h=15$ pixels at the end of the iterative procedure. This result tends to show that the highest subset size gives the best results in terms of tradeoff between spatial resolution and displacement resolution. This result must however be tempered by the fact that the procedure progressively diverges when the subset size increases, which means that some features of the noisy profile of the curve progressively significantly emerge, and can no longer be considered as a noise with an amplitude regularly distributed along the curve. Figure 5 illustrates this phenomenon in the case $h=23$ pixels. In this figure, it can be seen that the procedure diverges for $p_{\text {wave }} \approx 32$ pixels. 


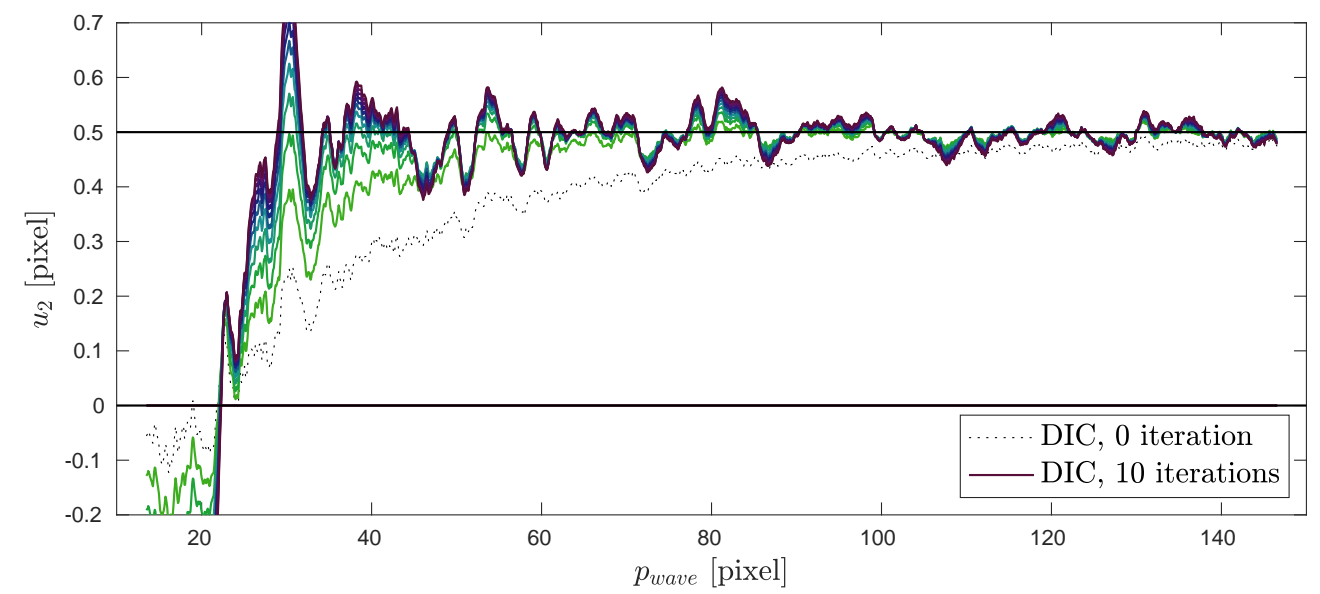

Fig. 5: Displacement obtained with speckle and DIC. Illustration of the divergence of the deconvolution procedure for $h=23$ pixels. The color of the lines turns from green to mauve as the iteration number increases. The procedure diverges for

$p_{\text {wave }} \approx 32$ pixels

Investigating all the curves obtained with all odd values of $h$ lower than 41 pixels does not permit to frankly see a value for $h$ which could be considered as a threshold, with convergence under this threshold and divergence above. $h=21$ pixels is the highest value without divergence with this example, but it seems to be dependent of the irregularities that impair the curve (some of them seem to be due to the effect of the random marking of the surface [29]). We will see in the following section that this effect can be attenuated for suitably filtering the effect of the deconvolution procedure in the Fourier domain.

\subsection{Correcting the bias induced by the Savitzky-Golay filter by threshold- ing the frequency during deconvolution}

As recalled in Section 2.2, the displacement returned by DIC performed pixelwise is, regardless of noise, equal to the actual and sought displacement convolved by a Savitzky-Golay filter, which is characterized by the subset size $h$ and the degree of the matching function. However the Savitzky-Golay filter exhibits a specific feature. For a first-degree matching function and from a certain value of the subset size on, the transfer function of this filter becomes negative for the highest frequencies involved in the reference displacement considered here. It means that for displacements corresponding to a pure sine of high-frequency (or small $p_{\text {wave }}$ ), such as in the left-hand side of the synthetic displacement field, DIC returns a displacement which is not only lower in amplitude, but also has the wrong sign. This property is illustrated and discussed in Ref. [5] (see Figure 3-b-c-e in this reference) and illustrated here in Figure 6, where it can be seen that this phenomenon occurs from $h=11$ pixels on. In this figure, we show the result along the axis of symmetry $\Delta$ of the map showing the convolution of the reference displacement in Figure 1 by a Savitzky-Golay filter of increasing width $h$. It can be seen that since we deconvolve the displacement field returned by DIC, the negative displacements obtained for the highest frequencies in Figure 5 are caused by the negative value of the transfer function of the Savitzky-Golay filter. 


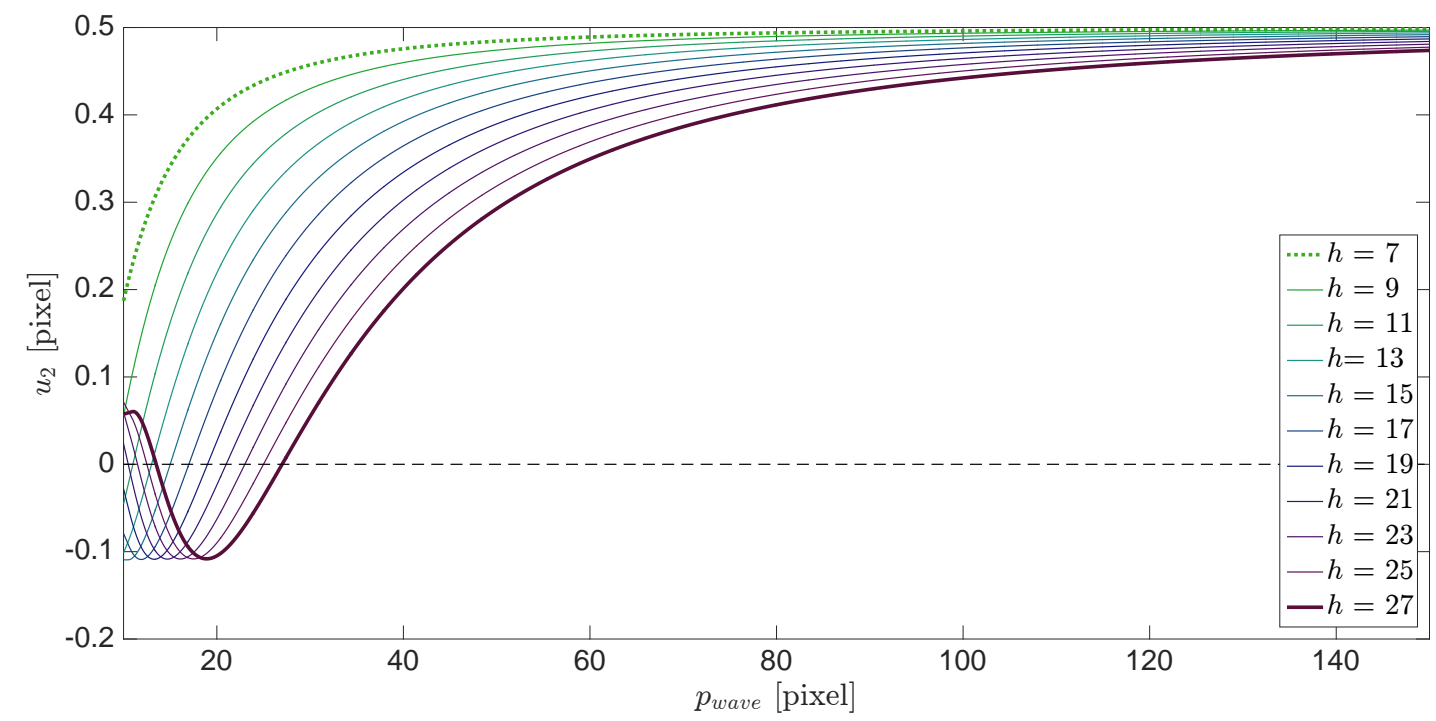

Fig. 6: Cross-section along the horizontal axis of symmetry $\Delta$ of the reference displacement in Figure 1 convolved by a Savitzky-Golay filter of increasing width $h$. The color of the lines turns from green to mauve as the subset size $h$ increases.

This issue is addressed here by applying the deconvolution procedure only for the frequencies lower than a certain threshold value denoted by $f_{\max }$. This is possible because the operations involved in the deconvolution procedure are performed in the Fourier domain. In this domain, one can easily perform deconvolution only within a window whose size depends on the threshold value $f_{\max }$. It is interesting to note that such an approach has been used in fluid mechanics for instance, and is known as soft deconvolution [25]. Figure 7-a shows the effect of this thresholding on the displacement returned by DIC along $\Delta$ for various values of $f_{\max }$. It can be seen that only the part of the curve concerned by the frequencies lower than $f_{\max }$ are impacted by deconvolution. The arrow designates the particular case characterized by $f_{\max }=1 / 40[\text { pixel }]^{-1}$. It can be observed that the part of the curve defined by $p_{\text {wave }}<40$ pixels is not affected by deconvolution, while the other part is. Considering a rectangular window in the Fourier domain may potentially lead a Gibbs effect to appear, which manifests itself by ringing artefacts in the resulting strain map. This phenomenon can be avoided by using a smoother window (as a Tukey window) instead of a rectangular one in the Fourier domain, with a width equal to the width of the rectangular window. A Tukey window is characterized by a dimensionless parameter $r$ lying between 0 and 1 . This window becomes a rectangular window with $r=0$ and a Hann window with $r=1$. Various preliminary tests (not detailed here) led us to choose $r=0.2$ throughout this study, in particular when plotting the curves shown in Figure 4 above. 
a- by using a rectangular window in the Fourier domain

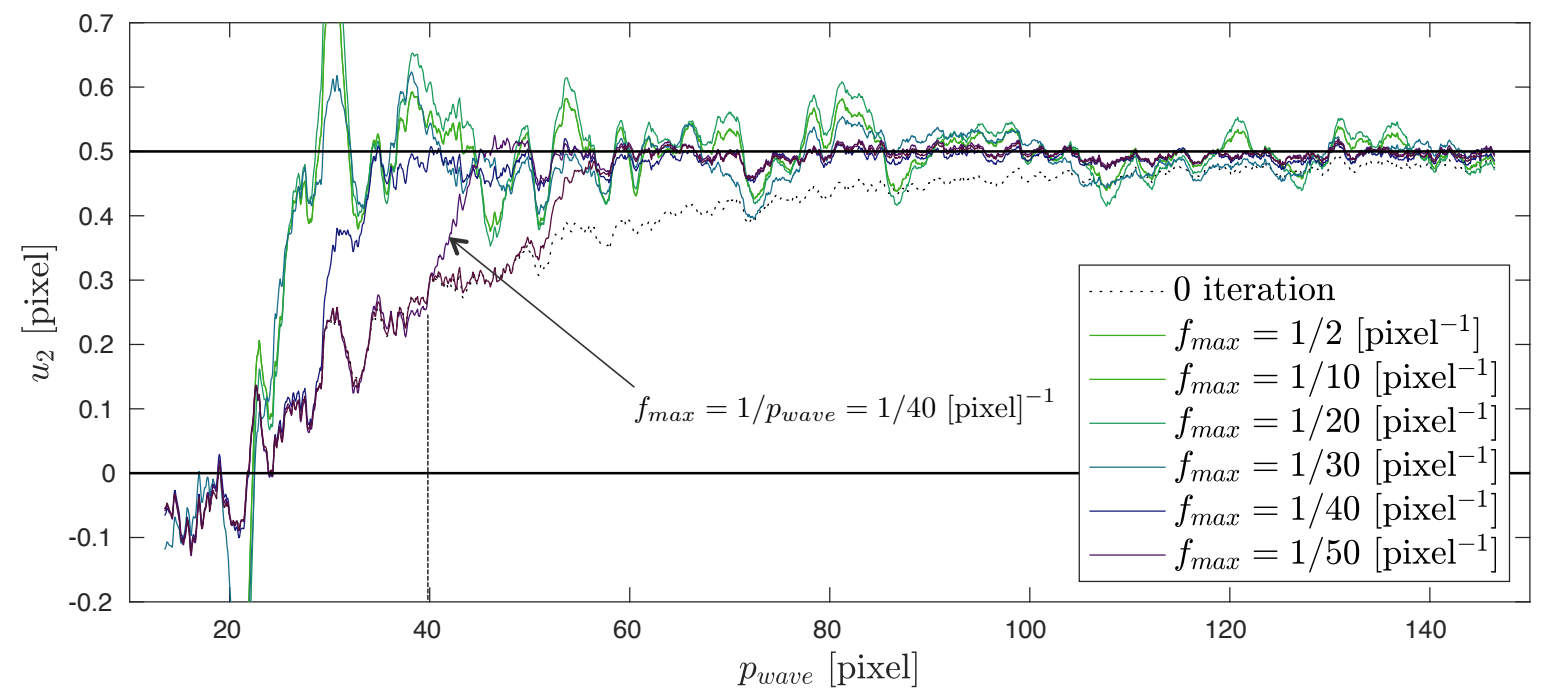

b- by using a Tukey window $(r=0.2)$ in the Fourier domain

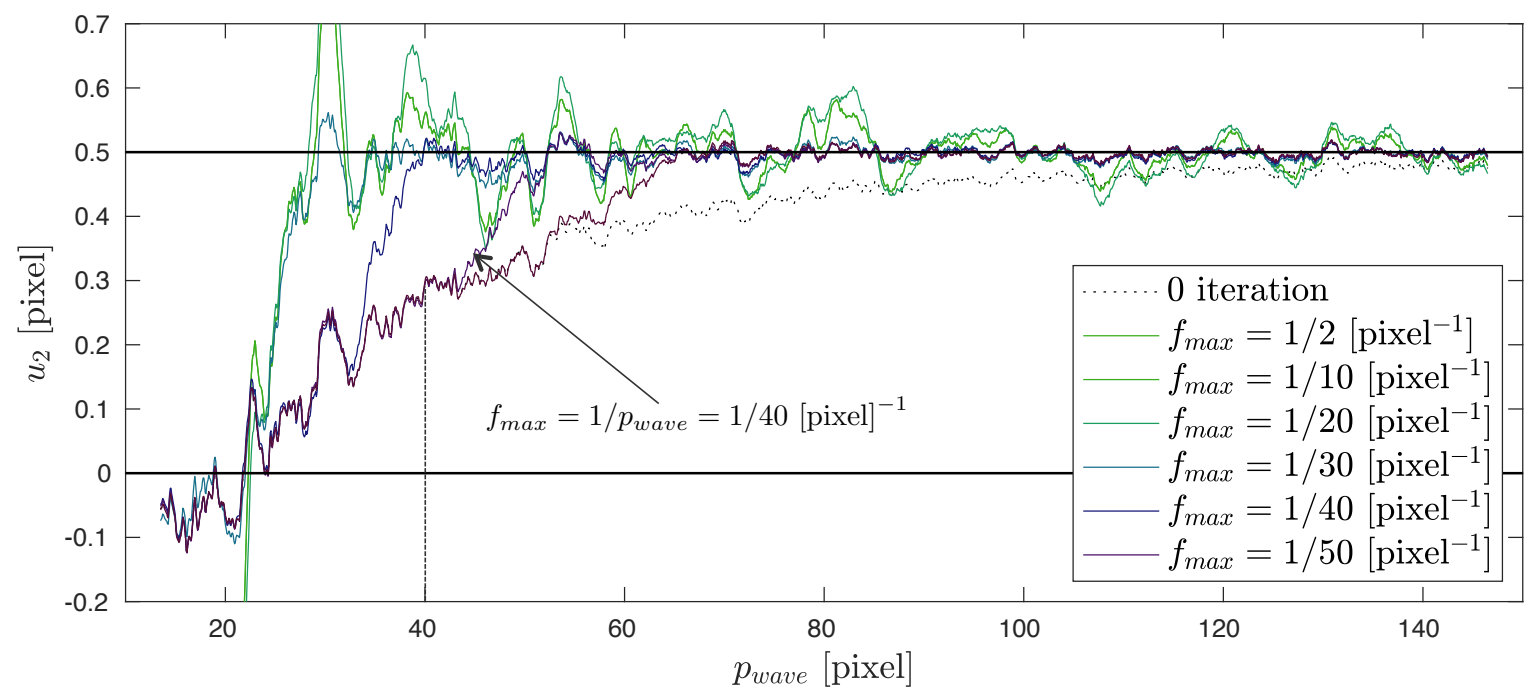

Fig. 7: Displacement obtained with speckle and DIC. Cross-section of the displacement along $\Delta$ obtained with the deconvolution procedure for $h=23$ pixels applied only for the frequencies lower than a variable threshold value $f_{m} a x$. The color of the lines turns from green to mauve as $f_{m} a x$ decreases. Illustration of the particular case $f_{\max }=1 / 40 \mathrm{pixel}^{-1}$ : on the left-hand side of $p_{\text {wave }}=1 / f_{\max }=40$ pixels, the curves obtained with and without deconvolution are the same. They become different under this threshold value because deconvolution impacts only the frequencies lower than $f_{\max }$, thus the periods $p_{\text {wave }}$ greater than $1 / f_{\max }$. Applying a Tukey window in the Fourier domain prevents from possible Gibbs effect. This slightly decreases the threshold value of the frequency under which deconvolution is applied, as can be seen in Figure 7-b.

The idea is therefore to adjust the threshold value for the frequency in such a way that only the part of the curve characterized by a positive displacement is affected by deconvo- 
lution. This procedure is expected to avoid the magnification of the errors obtained for the highest frequencies, when the sign returned by DIC is wrong. The value of $p_{\text {wave }}$ zeroing the displacement for a given subset size $h$ can be found numerically (this is the intersection between the horizontal axis and the representative curve of $h$ in Figure 6). Ref. [30] gives approximate values of the nominal normalized cutoff frequency ( $3 \mathrm{~dB}$ down), but it seems that no such expression is available to find the frequency zeroing the displacement in the present application. Interestingly, we empirically found that $\frac{1}{h}$ was a good approximation of this quantity. This threshold value being found, it is then used to define, in the Fourier domain, the size of the window within which the deconvolution procedure is applied. The result obtained by applying this procedure is shown in Figure 8 for the same value of $h$ as that used in Figure 5 to see the improvement brought about by this procedure.

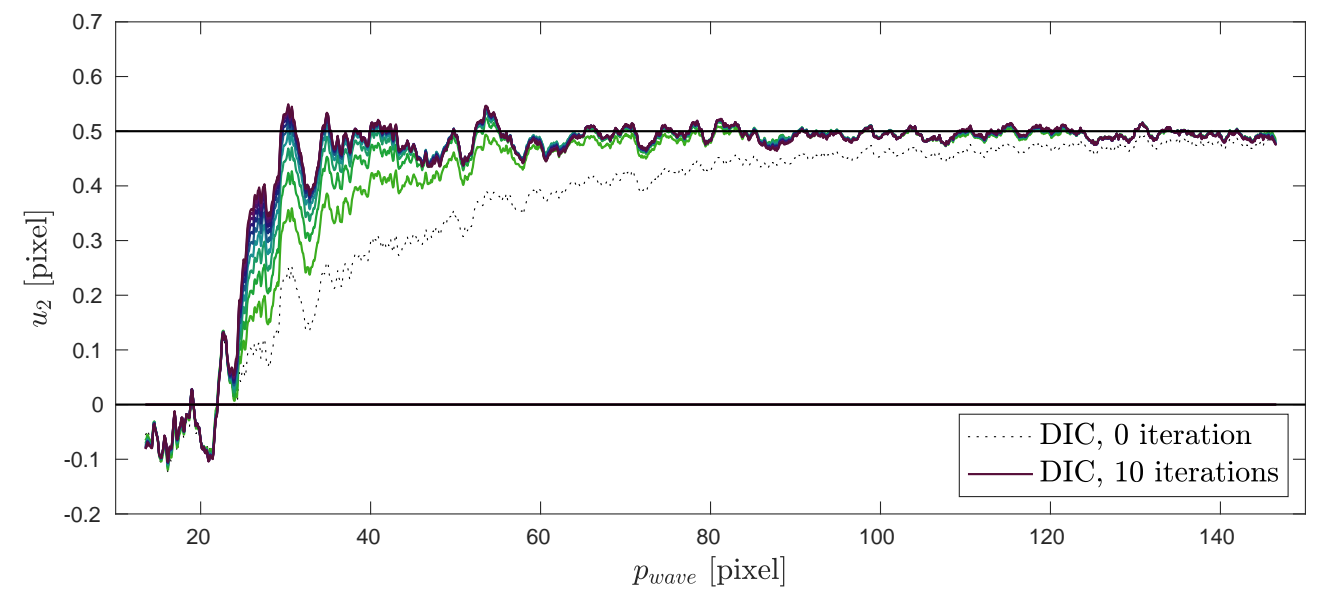

Fig. 8: Displacement obtained with speckle and DIC, for a subset size $h=23$ pixels. Crosssection along $\Delta$ of the displacement obtained with the deconvolution procedure applied only for the frequencies lower than a threshold value $f_{\max }=\frac{1}{h}$. The color of the lines turns from green to mauve as the iteration number increases.

It can be seen that applying this thresholding now not only keeps the displacement field unchanged above a certain frequency (thus on the left-hand side of the figure) but also strongly limits the significant fluctuations observed in Figure 5, and thus causes now the procedure to converge in this case, while the procedure diverges without thresholding. This illustrates that this thresholding step should be used systematically when applying the deconvolution procedure with DIC. As in the preceding section, it is however difficult to precisely determine a value of the subset size $h$ beyond which deconvolution applied only for the frequencies lower than $\frac{1}{h}$ converges. It can only be said that this threshold value for $h$ is greater with thresholding than without thresholding.

\subsection{Remark on the deconvolution of strain components}

We only discussed so far about the deconvolution of displacement or phase maps. It is however important to mention that strain maps obtained by differentiating displacement fields affected 
by convolution are also equal to their true counterparts convolved by the same kernel as that affecting the displacement. Indeed, since for any function $f$ and kernel $w$, we have $(w * f)^{\prime}=w * f^{\prime}$, where symbol' denotes derivation, we can deduce

$$
\begin{aligned}
\widetilde{\varepsilon}_{i j} & =\frac{1}{2}\left(\frac{\partial \widetilde{u}_{i}}{\partial x_{j}}+\frac{\partial \widetilde{u}_{j}}{\partial x_{i}}\right) \\
& =\frac{1}{2}\left(\frac{\partial\left(w * u_{i}\right)}{\partial x_{j}}+\frac{\partial\left(w * u_{j}\right)}{\partial x_{i}}\right) . \\
& =w * \varepsilon_{i j}
\end{aligned}
$$

It means that calculating the strains from deconvolved displacements also leads to deconvolved strains, and that the enhancement of the displacement maps discussed above also concerns the strain fields obtained by differentiation of the deconvolved displacement fields. In addition, strain fields being generally "less smooth" than displacement fields, higher frequencies are involved in their signature in the Fourier domain. Consequently, deconvolution should theoretically have a greater impact on strain maps than on displacement maps. Examples of deconvolution applied on strain maps are given in the last section of the paper.

\subsection{Conclusion}

In conclusion of this section, it can be said that:

- the deconvolution procedure proposed here improves the metrological performance of DIC in terms of tradeoff between spatial resolution and measurement resolution;

- a limitation of the procedure is that it progressively diverges as the subset size $h$ increases beyond a certain threshold value, which is however difficult to clearly define;

- the latter effect is attenuated by applying the deconvolution procedure only for frequencies lower than a threshold value of the frequency equal to the inverse of the subset size $h$.

\section{Minimization of the optical residual in the Fourier domain: checkerboard and LSA}

The objective here is the same as that of the preceding section, but now with LSA applied on checkerboards. The first point is to observe the positive effect of deconvolution. This is made by considering $\sigma=p=6$ pixels for the standard deviation of the Gaussian window used in LSA (see Equation 29 in Appendix 1). The Hessian components of the phases are obtained by differentiating the actual phases by convolution, by using the same settings as those adopted for DIC. Figure 9 shows the cross-section along the horizontal axis of symmetry $\Delta$ of the displacement field returned by LSA. Up to 20 iterations are applied in the deconvolution procedure. It is worth remembering that the artificial checkerboard images considered here are affected by the same type of noise as that affecting the artificial speckle images considered above for DIC. 


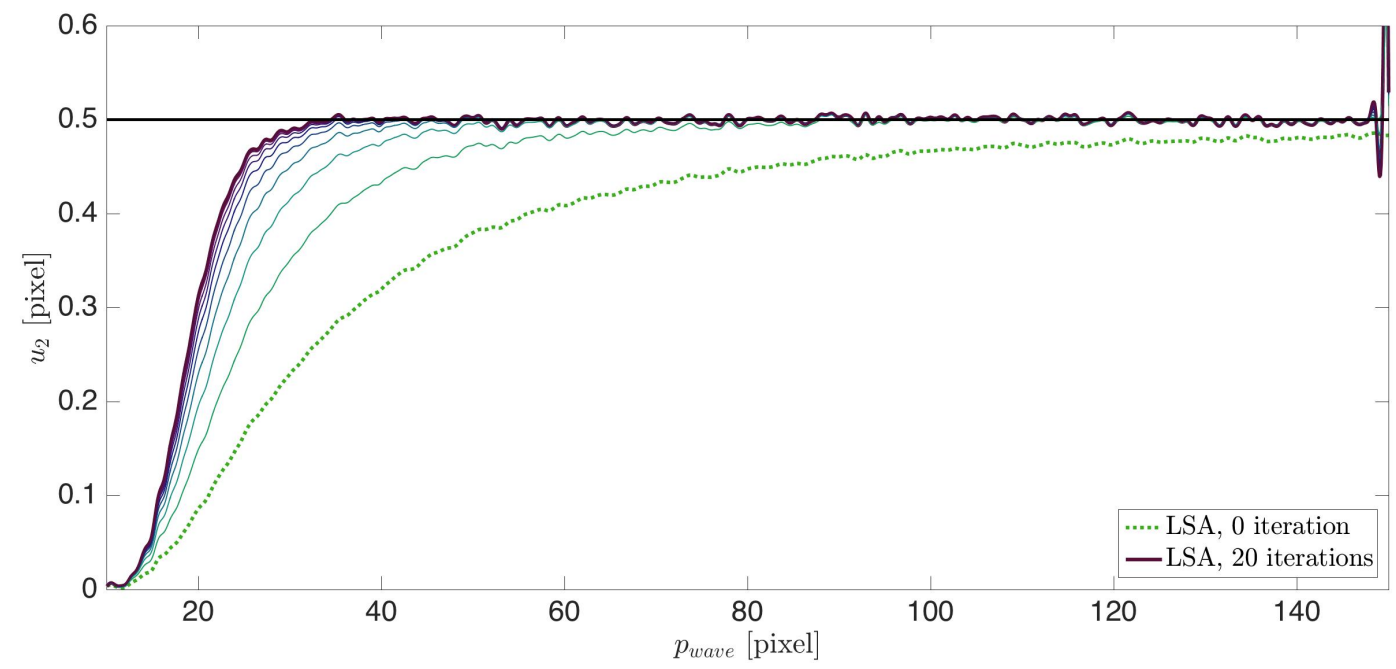

Fig. 9: Displacement obtained with checkerboard and LSA. Checkerboard images are affected by the same type of noise as that impairing the speckle pattern in Section 5. Cross-section along $\Delta$ of the displacement map returned by the deconvolution procedure during the first 20 iterations. The color of the lines turns from green to mauve as the iteration number increases.

Several remarks can be drawn from the curves depicted in this figure.

- As for DIC, the positive impact of the deconvolution procedure is clearly visible since the curves rapidly tend to the reference displacement $\left(u_{2}=0.5\right.$ pixels) up to a certain value of the period $p_{\text {wave }}$. In the following, we will therefore systematically perform 10 iterations. Less iterations are needed to converge with lower values of $\sigma$, so this number is conservative.

- The strong fluctuations on the right of the curves are caused by edge effects, where both the phases and the displacements are not correctly estimated.

- The checkerboard images are noisy and this noise propagates up to the displacement field. As for DIC, the same features are reproduced throughout the profile of the curves when considering results given by classic LSA (labelled "0 iteration" in this figure) and when applying the deconvolution procedure. $\sigma_{u}^{\mathrm{LSA}}$ and $\ell_{\lambda}^{\mathrm{LSA}}$ being obtained with the same procedure as for DIC, we can deduce $\alpha_{\lambda}^{\mathrm{LSA}}$ as the product between these two quantities. Figure 10-a represents $\alpha_{10}^{\mathrm{LSA}}$ and $\alpha_{20}^{\mathrm{LSA}}$ throughout the iterative procedure for two values of $\sigma$, namely $\sigma=p=6$ and $\sigma=p / \sqrt{2}=4.26$ pixels. The former is the pitch along the natural axes of symmetry $x_{1}$ and $x_{2}$ of the checkerboard, while $p$ is the pitch along the bisectors of the checkerboard. As in the case of DIC, $\alpha_{10}^{\text {LSA }}$ and $\alpha_{20}^{\mathrm{LSA}}$ regularly decrease and rapidly stabilize. This proves that the deconvolution procedure really improves the metrological performance of LSA in terms of trade-off between spatial resolution and measurement resolution. $\lambda$ is fixed arbitrarily in turn to $10 \%$ and $20 \%$ but it can be seen that the same conclusion can be drawn for these two values of $\lambda$. 
- In Figure 10-a, it can be seen that for classic LSA ("0 iteration") and for a given value of $\lambda$, the values of $\alpha_{\lambda}^{\text {LSA }}$ obtained for $\sigma=p=6$ and $\sigma=p / \sqrt{2}=4.26$ pixels are the same, which confirms again that $\alpha_{\lambda}^{\mathrm{LSA}}$ is a constant parameter independent of $\sigma[4,28,16]$. Second, it can be seen that for a given value of $\lambda, \alpha_{\lambda}^{\mathrm{LSA}}$ in the case $\sigma=p$ gives results which are significantly better than in the case $\sigma=p / \sqrt{2}$. The spatial resolution $\ell_{\lambda}^{\mathrm{LSA}}$ throughout the iterative procedure, plotted in Figure 10-b, gives the reason. The phenomenon observed for DIC is amplified here, since the spatial resolution $\ell_{\lambda}^{\mathrm{LSA}}$ becomes lower (thus better) for the greatest window of the two (in Figure 10-b, the curves for $\sigma=p / \sqrt{2}$ and $\sigma=p$ intersect). Since at the same time, the noise level is always lower for the greatest window, the product between the two quantities, which defines parameter $\alpha_{\lambda}^{\mathrm{LSA}}$, is lower for the greatest value of $\sigma$ at the end of the iterative procedure. It means that in practice, a window of standard deviation $\sigma=p$ should systematically be chosen when the procedure is used since one has at the same time the lowest noise level, the lowest spatial resolution, and a bias which is almost null for frequencies of the signal lower than a certain threshold value. This value has been found here by seeking $\ell_{1}^{\mathrm{LSA}}$ (which means that $1 \%$ error is permitted on the amplitude of the signal returned by the technique) and taking its inverse. We found $\ell_{1}^{\mathrm{LSA}}=52.1$ pixels for $\sigma=p / \sqrt{2}$, and $\ell_{1}^{\mathrm{LSA}}=29.9$ pixels for $\sigma=p$. Interestingly, the latter quantity is slightly lower than the apparent size of the Gaussian window according to the "3- $\sigma$ rule" [31]. This rough estimate of the size is equal to $2 \times 3 \times p=2 \times 3 \times 6=36$ pixels. It means that all components of the signal, whose period is slightly lower than the apparent size of the window, are progressively lost, which seems logical. A good point is that, on the contrary, the components of the signal whose period is greater than a threshold value slightly lower than the size of the window, are returned without any systematic error. This illustrates the strength of the method.
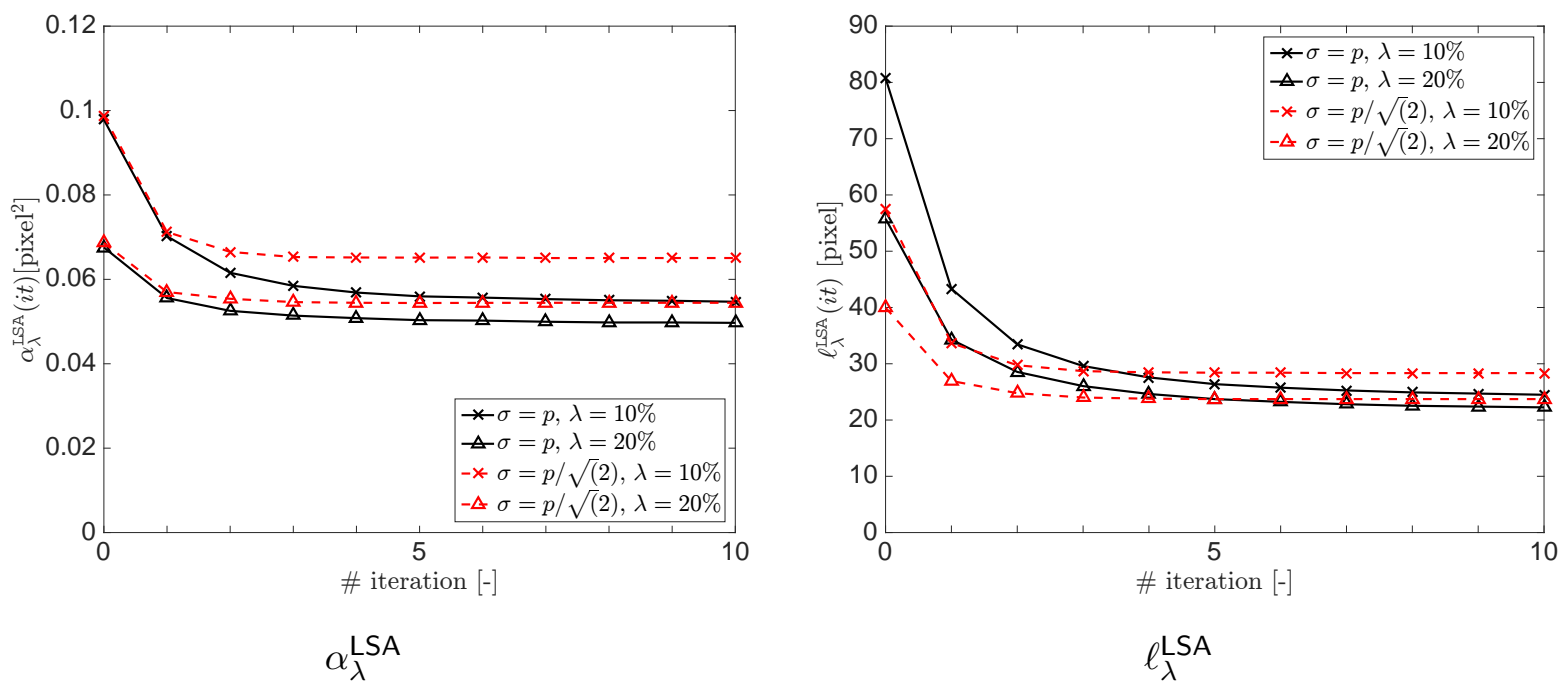

Fig. 10: $\alpha_{\lambda}^{\mathrm{LSA}}$ and $\ell_{\lambda}^{\mathrm{LSA}}$ during the first 10 iterations for $\sigma \in\{p / \sqrt{2}, p\}$ and $\lambda \in\{10 \%, 20 \%\}$.

- Contrary to what happens with DIC, the sign of the displacement returned by LSA does not change for the highest frequencies (see the left-hand side of the curves in Figure 9). The same remark holds whatever the value of $\sigma$. This is due to the fact that the Fourier 
transform (and thus the transfer function) of the Gaussian function used in LSA is also a Gaussian function. It is therefore always positive. No thresholding of the frequency is therefore applied in the simulations presented here. However, we will see in Section 7 below that thresholding the frequency during deconvolution enables us to reduce the noise level in the strain maps obtained with LSA. The price to pay is that the actual details are also smoothened in proportion.

- Finally, we investigate the influence of the value of $\sigma$ on the convergence of the deconvolution procedure. Figure 11 shows the displacement along $\Delta$ for various values of $\sigma$. It can be seen that the procedure converges for values of $\sigma$ lower or equal to the pitch $p$ of the checkerboard and diverges for greater values. Indeed divergence is observed for the lowest pitches $p_{\text {wave }}$ of the sinusoidal displacement, thus for the highest frequencies. This confirms that $\sigma=p$ is certainly the best choice when processing the images with LSA and applying the deconvolution procedure.

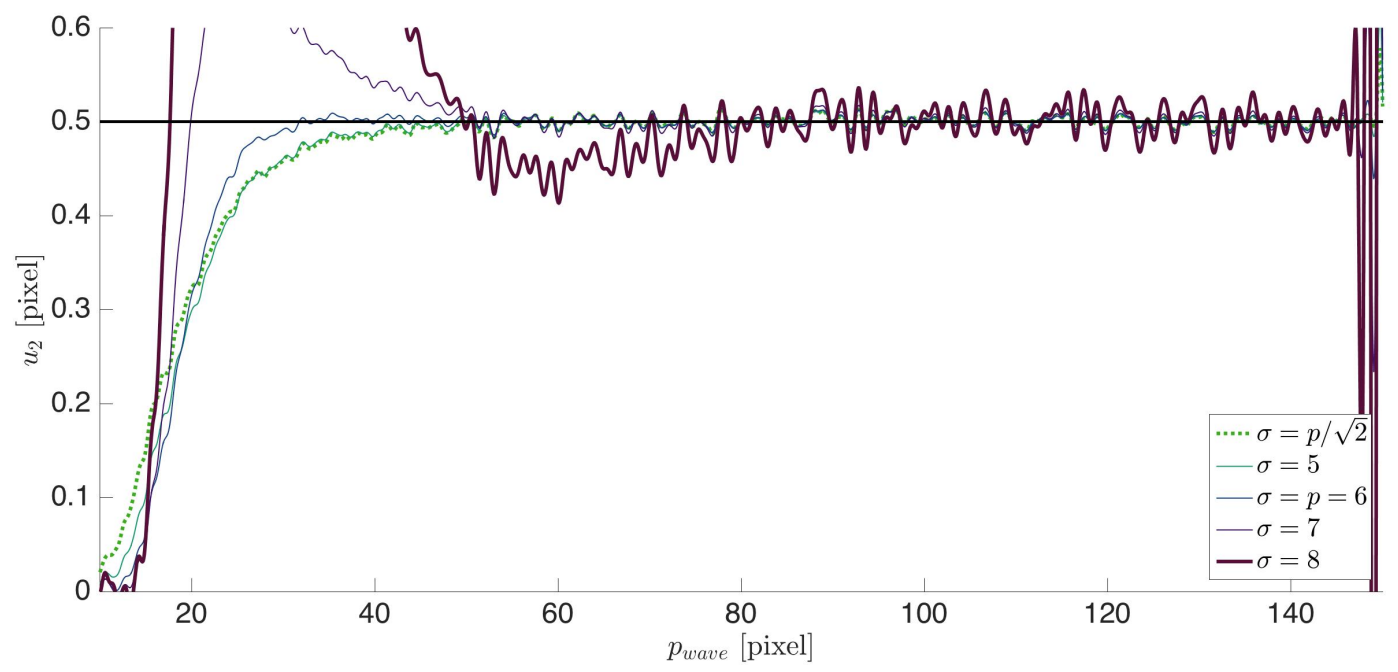

Fig. 11: Displacement obtained with checkerboard and LSA. Cross-section along $\Delta$ of the displacement map returned by the deconvolution procedure after 10 iterations and for various values of $\sigma$. The color of the lines turns from green to mauve as $\sigma$ increases.

In conclusion of the two preceding sections, we show in Figure 12 the displacement fields retrieved after applying DIC and LSA followed or not by the deconvolution procedure. The optimized conditions defined above are used for each technique. Hence, we have $h=21$ pixels for DIC and $\sigma=p=6$ pixels for LSA. 10 iterations are performed in each case. The phenomena discussed above are clearly visible. On the very left, the signal is lost because the frequency of the displacement is too high. Then the effect of deconvolution reaches its maximum. Finally, when going to the right, thus to the lowest frequencies of the displacement, the effect of deconvolution progressively vanishes. Indeed the quantity to be added to the displacement fields obtained without deconvolution becomes smaller to get rid of the bias since the frequency of the signal diminishes. 


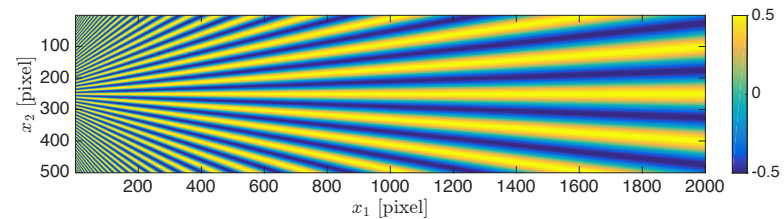

(a) Reference displacement field

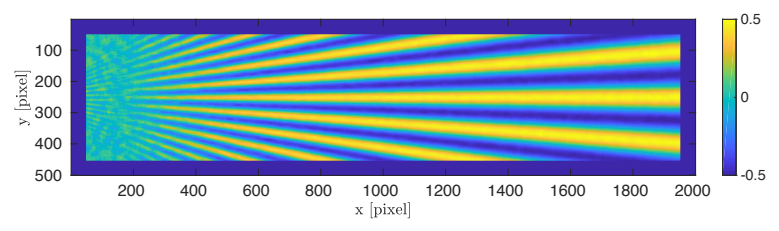

(c) DIC, 0 iteration

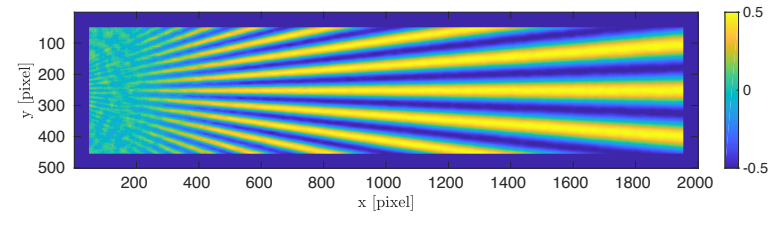

(e) DIC, 10 iterations

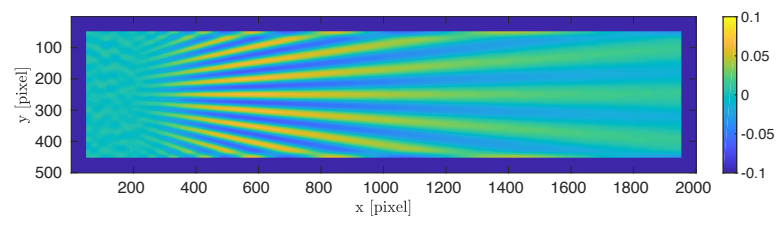

(g) DIC, difference between 10 and 0 iterations

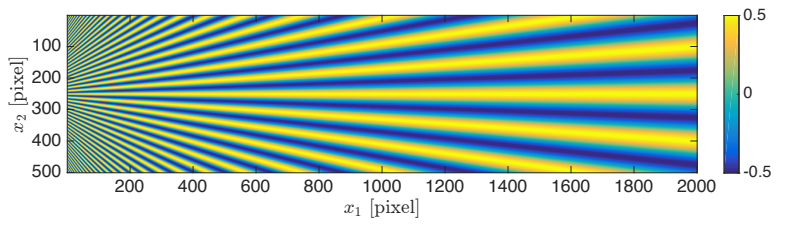

(b) Reference displacement field

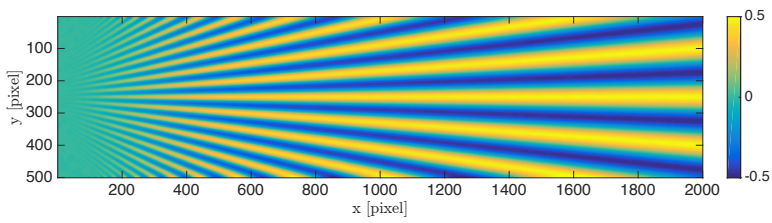

(d) LSA, 0 iteration

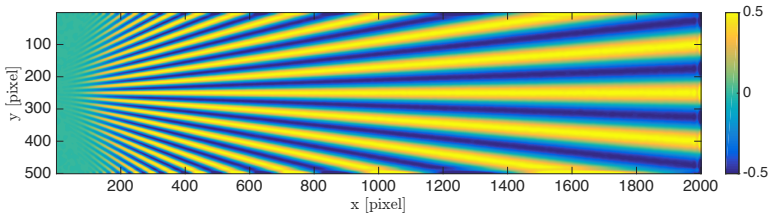

(f) LSA, 10 iterations

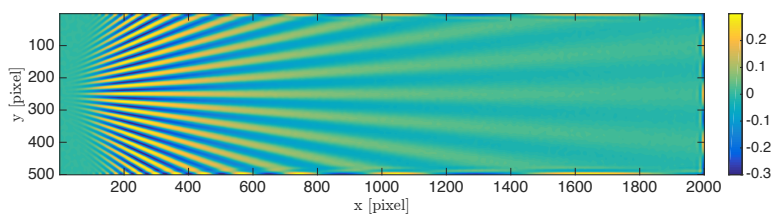

(h) LSA, difference between 10 and 0 iterations

Fig. 12: Effect of deconvolution on the displacement field. DIC performed with $h=21$ pixels, LSA with $\sigma=6$ pixels.

\section{Application to a real case}

The deconvolution procedure is now applied on real data. We choose here a case for which significant strain gradients occur in the map. This example deals with wood, as shown in Figure 13 where the specimens used with DIC and LSA are represented. They are made of pine and cut is such a way that the annual rings due to earlywood and latewood appear on the front face of the specimens. The dimensions of the specimens are $50 \times 35 \times 15 \mathrm{~mm}^{3}$. A speckle is spray-painted on the first specimen for DIC. For LSA, a checkerboard of pitch $p=0.2 \mathrm{~mm}$ is deposited on the front face of the second specimen using the procedure described in [32]. A 12-bit CCD Sensicam camera featuring $1376 \times 1040$ pixels is used to capture the images of the front face of both specimens during a compression test applied along the vertical $\left(x_{2}\right)$ direction. In the checkerboard image, the pitch is equal to 5.77 pixels. It is close to the optimal value $\mathrm{p}=6$ pixels (see Section 4.2 ). The principal axes of the checkerboard are slightly rotated with respect to the rows of pixels of the camera in order to avoid the effect of aliasing in the strain maps, as proposed in [33]. These specimens were subjected to a compression test. The objective here is to examine the effect of the deconvolution procedure on one of the strain 
maps obtained with each of the two techniques (DIC and LSA). The strain level is higher in the map chosen to illustrate deconvolution with DIC, the sensitivity to noise being expected to be higher with this technique according to the results found in the preceding sections.
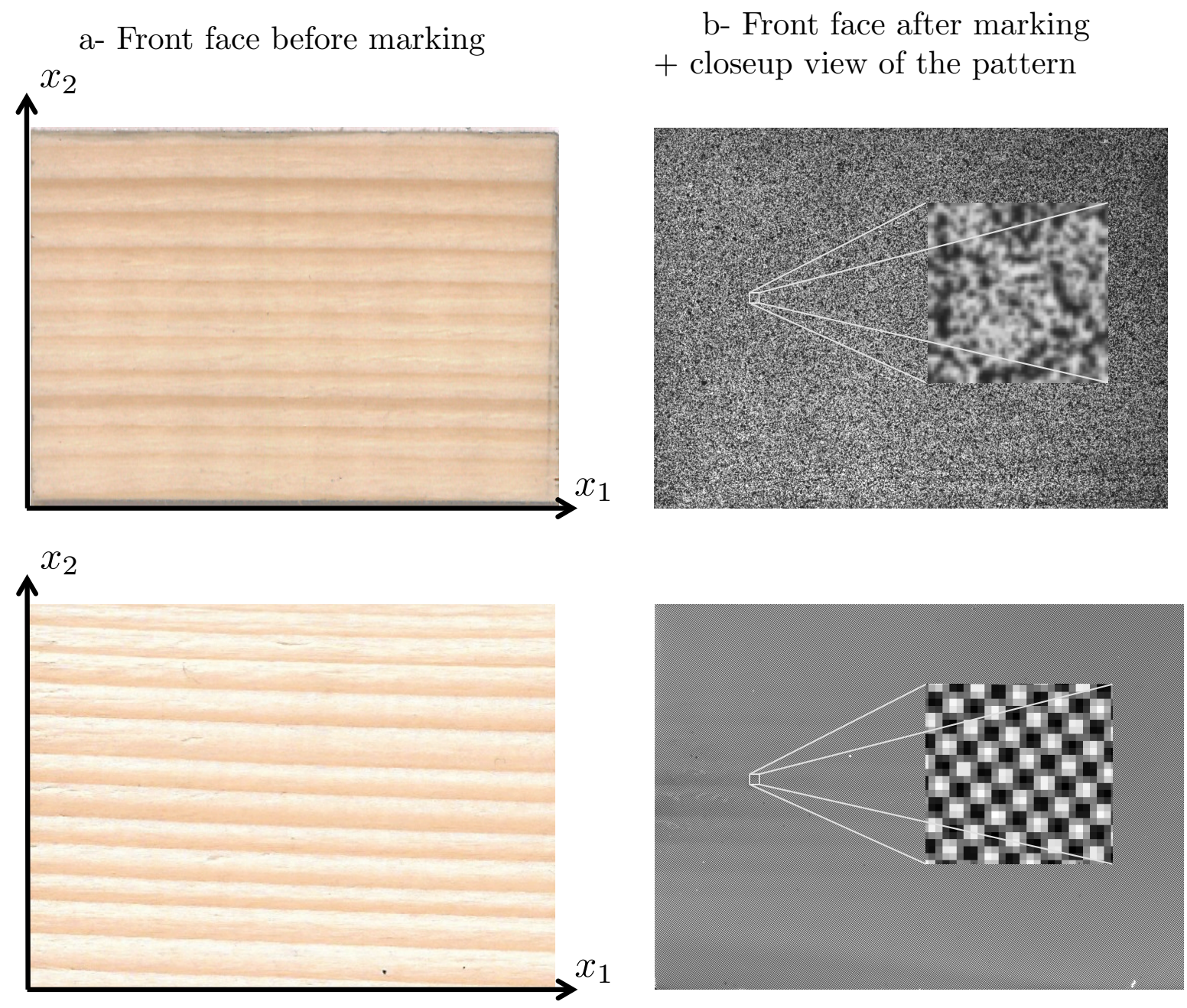

Fig. 13: Wood specimens used for the compression test. Top: specimen marked with a speckle pattern for DIC. Bottom: specimen marked with a checkerboard pattern for LSA (the closeup views are not to scale)

\subsection{Results: specimen marked with a speckle}

We show in Figure 14-a a typical example of $\varepsilon_{22}$ strain map obtained during this test. A subset size $h=21$ pixels is used here in DIC. Applying the deconvolution procedure with 10 iterations gives the map shown in Figure 14-b. Deconvolution is applied on a bigger map in order to avoid edge effects, which are due to the fact that the second derivatives along a certain direction cannot be determined along the border of the specimen if this border is not parallel to this direction. This bigger map is artificially created from the initial one by 
copying and pasting, by symmetry along the edges, a portion of the first map. Hence the edge effect impairs this big map during deconvolution, not the small one from which it is defined and which is located at the center.

Figure 14-c shows the difference between the $\varepsilon_{22}$ maps obtained before and after deconvolution. It can be seen that the amplitude of the wave in the maps due to the presence of earlywood and latewood really increases after deconvolution, which illustrates the positive impact of deconvolution. The diameter of the red circles superimposed on these figure is equal to the spatial resolution. Its reduction (thus its improvement) after deconvolution is noticeable. Another point is that Figure 14-c is rather smooth. It means that the highest frequencies of the noise are the same before and after deconvolution. This is logical since we applied here the deconvolution procedure only for the frequencies lower than the cutoff frequency $\frac{1}{h}$, as justified in Section 5.2. The highest frequencies impairing the strain map in Figure 14-a are therefore kept unchanged in Figure 14-b. A consequence is that the sharpest details in the strain map (those described by frequencies greater than $\frac{1}{h}$ ) are not enhanced by the deconvolution procedure. 

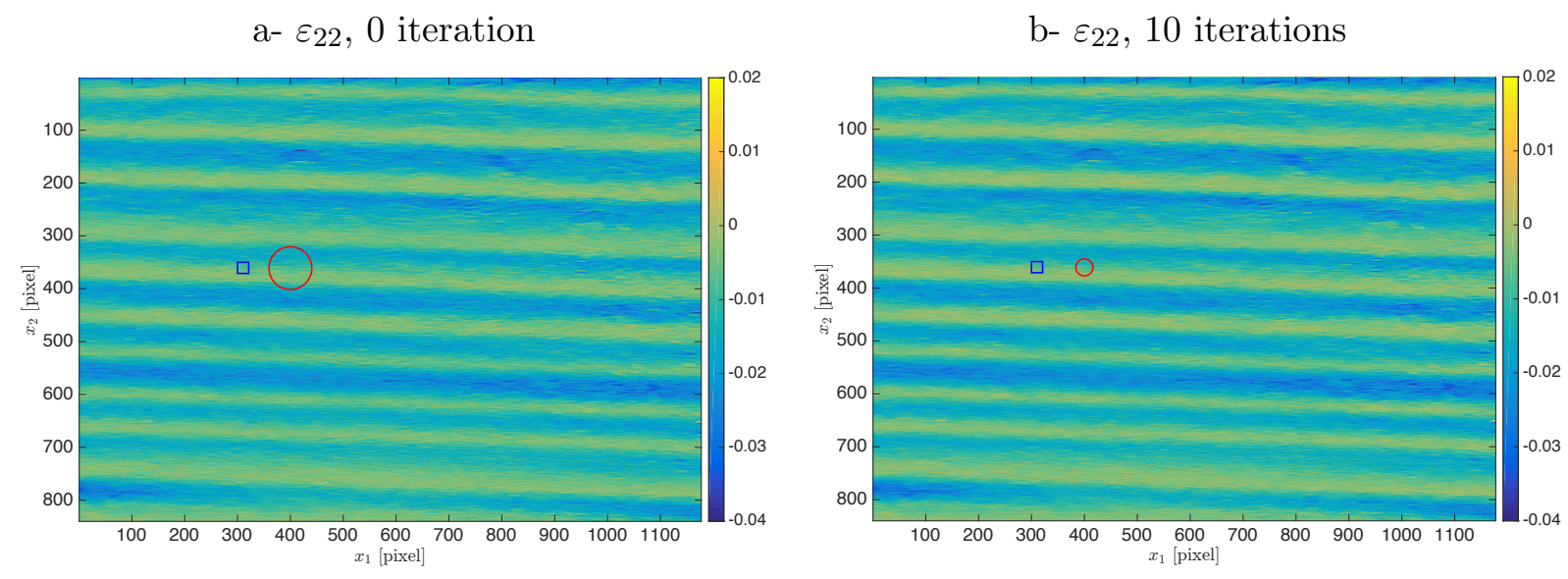

c- $\Delta \varepsilon_{22} \times 10^{-3}$

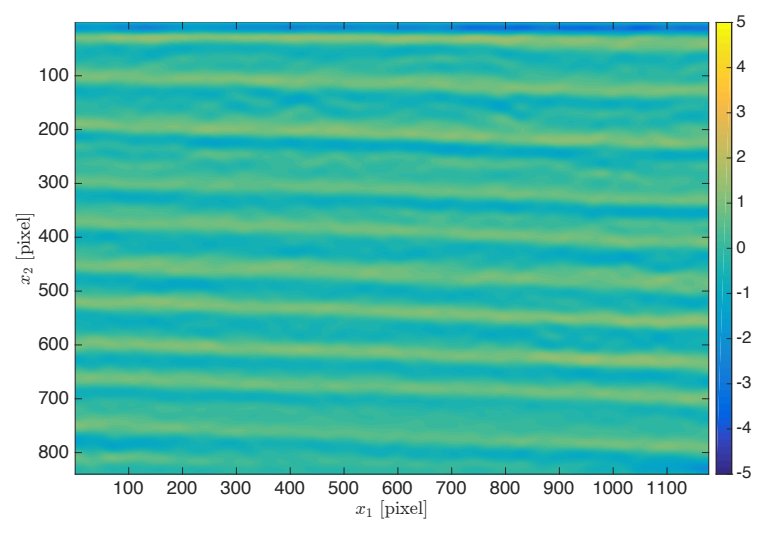

Fig. 14: Typical strain maps obtained with speckle and DIC during the compression test. Blue square: subset size. Diameter of the red circle: equal to the spatial resolution $\ell_{\lambda}$ for a bias $\lambda=10 \%$. a- DIC without deconvolution ("0 iteration"). b- DIC after deconvolution ("10 iterations"). c- difference.

\subsection{Results: specimen marked with a checkerboard}

The same discussion is proposed here for LSA. A typical example of $\varepsilon_{22}$ strain map given by classic LSA is shown in Figure 15-a. The value of $\sigma$ is $\sigma=p$ in this case. 10 iterations are used in the deconvolution procedure.

Figure 15-b shows the strain maps obtained after applying the deconvolution procedure. In this first example, we keep the edge effect to illustrate its visual impacts on the results if it is not removed. The difference between deconvolved and initial maps is represented in Figure 15d. It confirms that the strain bands are sharper after deconvolution. As expected, noise is slightly amplified after deconvolution. This effect can be attenuated by applying the same pre-filtering procedure as that described for DIC in Section 5.2. In this case, deconvolution is applied only for frequencies lower to the cutoff frequency considered when applying the prefiltering stage. The convolution kernel is, however, now a Gaussian function and no longer a Savitzky-Gollay filter, so the cutoff frequency is no longer the frequency for which a change of 
sign is observed in the displacement field discussed in Section 4. The displacement returned by LSA keeps indeed the same sign for any value of the frequency of the signal. The curves in Figure 9 show that the value of this cutoff frequency can reasonably be chosen as equal to $f_{\max }=1 / 10$ pixel $^{-1}$ in the case of LSA, this technique being unable to return any reliable information above this frequency. Figure 15-c shows the strain maps obtained by applying this procedure. The edge effect has also been suppressed by applying the same procedure as that described above for DIC. It can be seen that the noise in the strain map is lover than that observed in Figure 15-b. This is confirmed by considering in Figure 15-e the difference between deconvolved and initial strain fields.

Figure 16 shows the correction due to deconvolution expressed as a percentage of the initial strain value at any pixel. The two cases discussed above are considered here, namely by applying the deconvolution procedure $i$ - without any thresholding of the frequencies (Figure 16-a) and it- only for the frequencies lower than $f_{\max }=1 / 10$ pixel $^{-1}$ (Figure 16-b). It can be seen that the map is noisier on the left but the contrast is slightly lower on the right.

This difference between deconvolved and initial strain maps is clearer when plotting a vertical cross-section of these strain maps (see Figure 17-a). It is worth noting that only 3 or 4 iterations are needed to reach convergence in this example, no visible improvement being detected in this figure for higher numbers of iterations.

In such materials, an important point is to observe the difference in stiffness between earlywood and latewood. This difference in stiffness is reflected here by the difference in strain amplitude, which gives the peaks and valleys in Figure 17-a. It is clear that these peaks and valleys become shaper after deconvolution. In the closeup view shown in Figure 17-b, the difference in strain between a typical peak (point $A$ ) and valley (point $B$ ) is equal to $\Delta \varepsilon_{22}^{(0)}=2.82 E-03$ before deconvolution and $\Delta \varepsilon_{22}^{(15)}=3.88 E-03$ after deconvolution, which is non negligible $(+38 \%)$ if the objective is to assess the contrast in stiffness between earlywood and latewood. Applying the convolution procedure only for frequencies lower than $f_{\max }=1 / 10$ pixel $^{-1}$ gives the strain distribution shown in Figure 17-c, which is a crosssection of Figure 15-c along a vertical line. The strain map in Figure 15-c is slightly smoother than in Figure 15-b, but Figure 17-c shows the consequence, since the difference in strain between point $A$ and point $B$ is now equal to $\Delta \varepsilon_{22}^{(10)}=3.37 E-03$ after deconvolution $(+19.5 \%)$, which is lower than in the preceding case where deconvolution was applied for all the frequencies. Additional investigations are needed to elaborate the best strategy to get the most reliable strain distribution with the lowest noise. In particular, it is not sure that applying the deconvolution procedure only for the frequencies lower than a certain threshold value is the best choice. This leads to a lower noise but this also induces a loss of real information. If the goal is to identify material properties, applying for instance the Virtual Fields Method [34] leads to spatially average out the noise, and thus applying deconvolution without thresholding the frequency is perhaps better in this case. This should however be investigated with a dedicated study.

In conclusion, the results presented here illustrate in both cases the benefit brought about by deconvolution when measuring strain in case of high and localized strain gradients. 


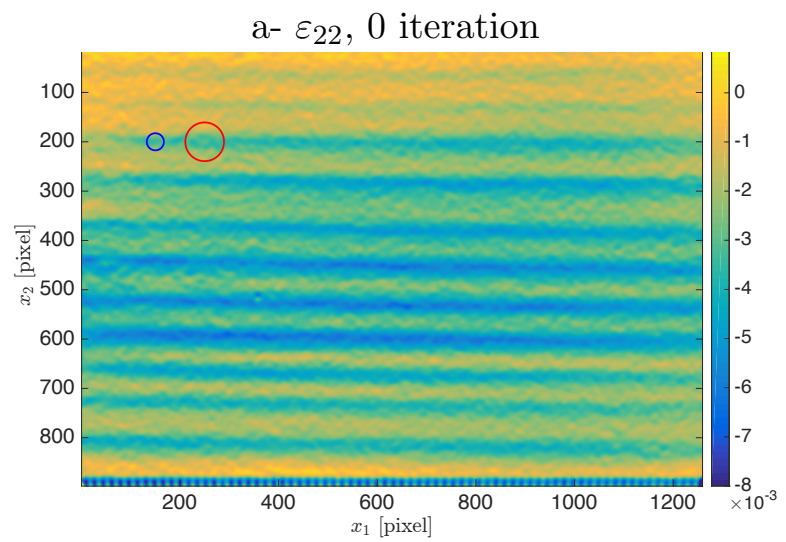

b- $\varepsilon_{22}, 10$ iterations without thresholding in the Fourier domain and with edge effect

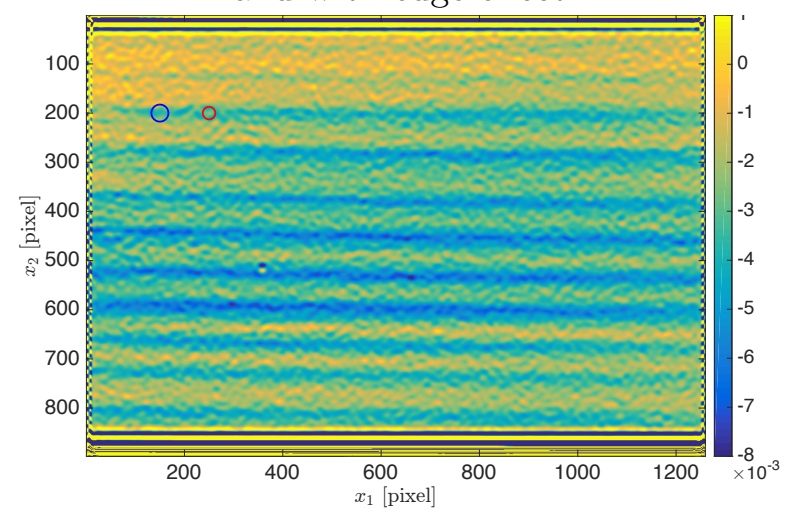

d- $\Delta \varepsilon_{22} \times 10^{-3}$ without

thresholding in the Fourier domain and with edge effect

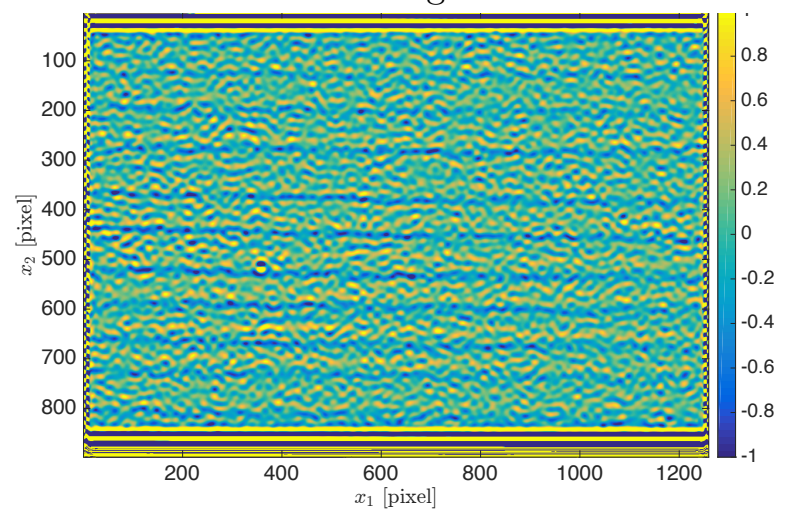

c- $\varepsilon_{22}, 10$ iterations with thresholding in the Fourier domain and without edge effect

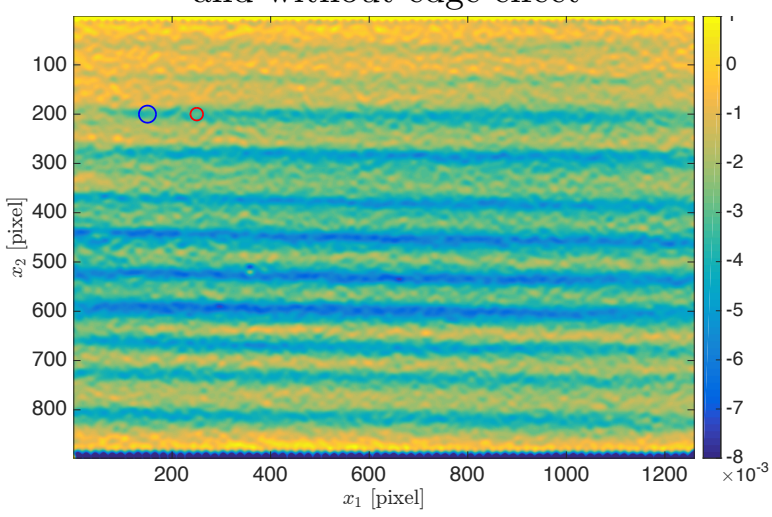

e- $\Delta \varepsilon_{22} \times 10^{-3}$ with

thresholding in the Fourier domain and without edge effect

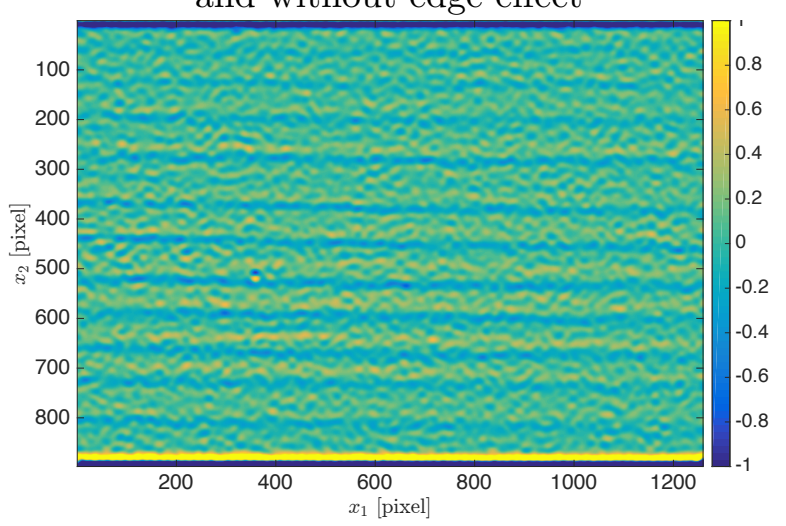

Fig. 15: Typical strain maps obtained with checkerboard and LSA during the compression test. Blue circle: size of the Gaussian window used in the WFT according to the $3-\sigma$ rule [31]. Diameter of the red circle: equal to the spatial resolution $\ell_{\lambda}$ for a bias $\lambda=10 \%$. Left-hand column: with edge effect due to deconvolution and without thresholding. Right-hand column: edge effect due to deconvolution removed and with thresholding, $f_{\max }=1 / 10$ pixel $^{-1}$ 

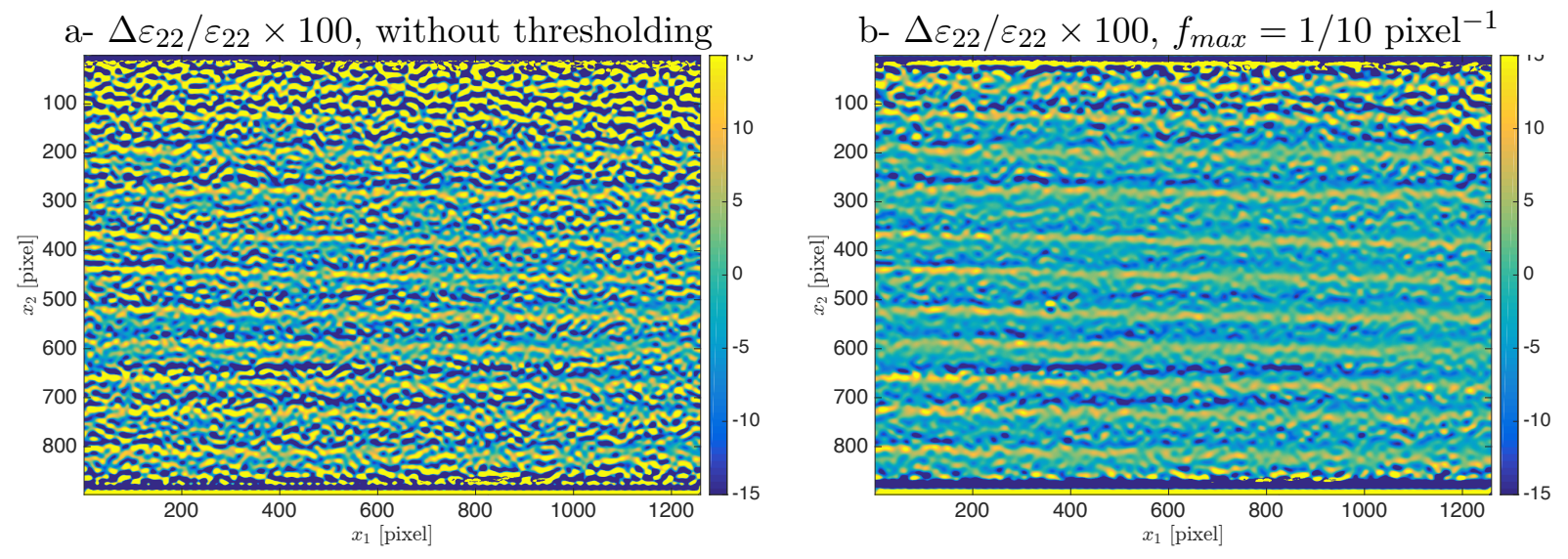

Fig. 16: Correction of the initial strain map as a percentage of the strain obtained before deconvolution. a- without thresholding b- with thresholding. Results given in $\%$ 
a- cross-section of the $\varepsilon_{22}$ distribution obtained during the iterative procedure without filtering.

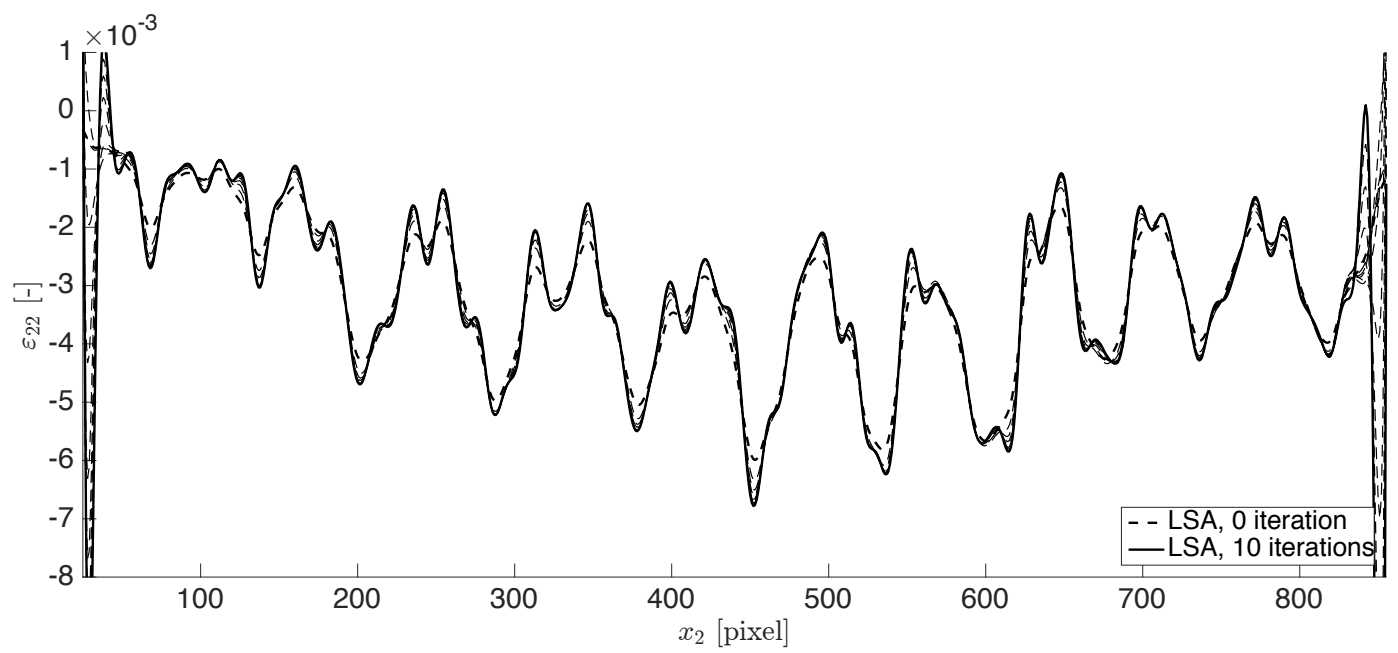

b- closeup view between $x_{1}=270$ pixels and $x_{1}=420$ pixels. Without filtering

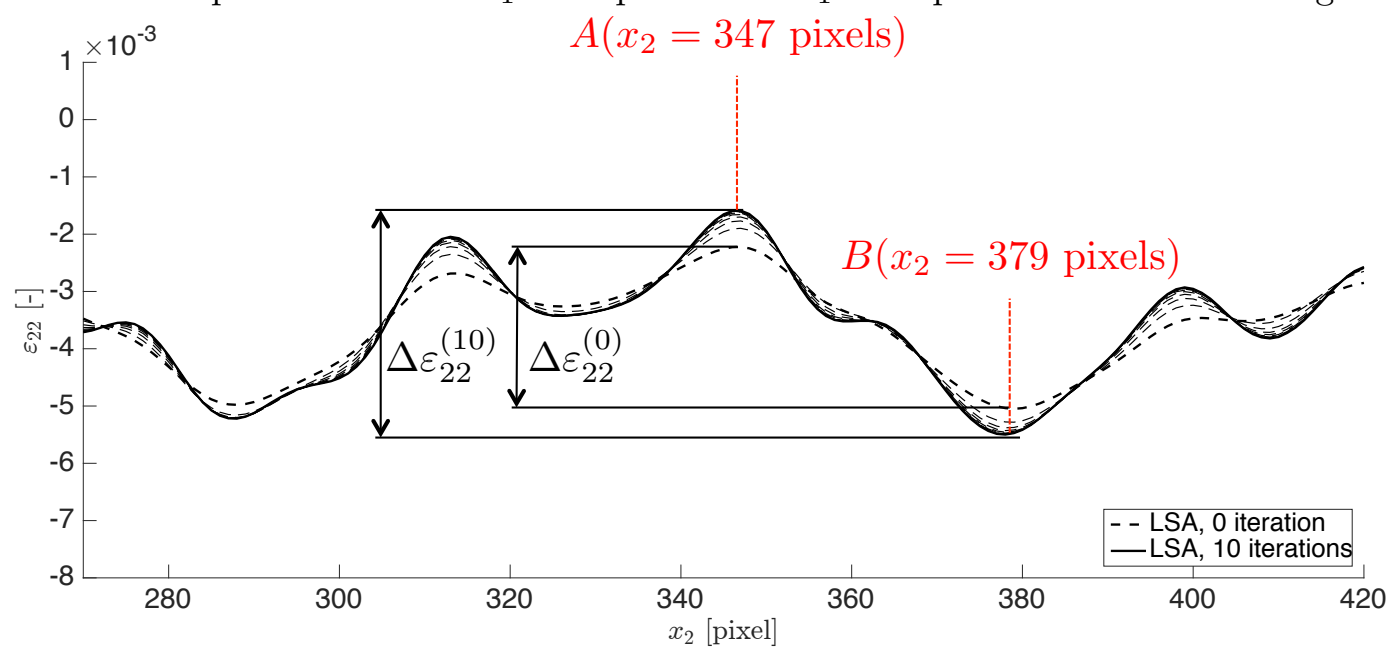

c- closeup view between $x_{1}=270$ pixels and $x_{1}=420$ pixels. With filtering

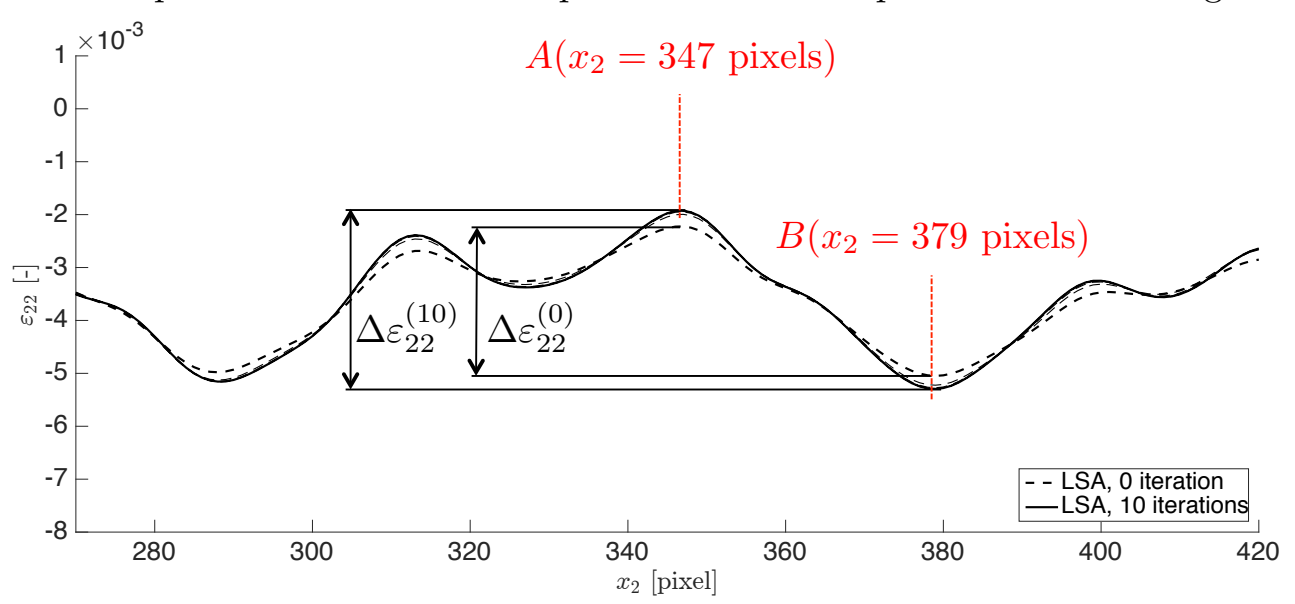

Fig. 17: Cross-section of the $\varepsilon_{22}$ distribution during the iterative deconvolution procedure, $x_{1}=500$ pixels. 


\subsection{Computation time}

The computational cost of deconvolution is briefly investigated here. It is worth remembering that deconvolution is a post-processing procedure, which is applied after using classic DIC or LSA. As a consequence, since only the definition of the kernel changes from one technique to another, the computational cost is nearly the same for both techniques for a given size of map to be deconvolved. The quantity which is deconvolved is however different for DIC and LSA since this is directly the displacement for LSA and the phase for LSA. The displacement is then deduced from differences between phases in the latter case, as explained in Section 2.3. It means that the number of maps to be deconvolved to obtain a displacement or strain map is theoretically twice higher for LSA than for DIC, and so the calculation time. The fixed-point algorithm used to obtain the displacement (see Equation 6) being non-linear, one cannot directly deconvolve the displacement field for LSA. This is possible only if the phases are directly subtracted, but the movement between the reference and current images is not compensated in this case, which is not recommended. To give an idea of the computation times, we consider as an example the case of the wood specimens discussed in Sections 7.1 and 7.2 above. Each iteration lasts about 1.48 second for strain maps obtained with DIC (the maps contain 989,857 pixels in this case), and 3.37 seconds for strain maps obtained with LSA (the maps contain 1,130,582 pixels in this case). The desktop computer used for these calculations is equipped with a $2 \times 8$-core Intel Xeon CPU E5-2620 v4 @ 2.10GHz and $64 \mathrm{~Gb}$ memory. The deconvolution algorithm was programmed in Matlab.

\subsection{Comparison with strain maps deconvolved with the Richardson-Lucy algorithm}

The reader may wonder which result would be obtained with an off-the-shelf deconvolution procedure. We propose here to answer this question by showing the results given by one of the most popular deconvolution algorithms in image processing, namely the Richardson-Lucy (RL) algorithm [35, 36]. This algorithm is iterative. It was developed for natural images, and the signal to be processed is necessarily positive since the denoised image is retrieved as a maximum likelihood estimation under a Poisson noise assumption. Although strain fields do not satisfy this assumption, it has been shown in [23] that this very simple procedure gives deconvolved strain fields that show equivalent or less spurious artifacts than methods relying on an accurate noise model such as Wiener filters. However the classic deconvolution algorithms, which are tested in [23], give reliable results only with a favorable signal-tonoise ratio. As an example, with the noise level in the images obtained with the camera used in [23], the strain components had an order of magnitude of several percents to have a successful deconvolution with the RL algorithm.

For the sake of comparison, let us observe what happens with the RL algorithm applied to the example discussed above. Since we deal here with strain components which can potentially be negative (this is the case here), the strain map obtained by classic LSA to be deconvolved must first be transformed to become strictly positive, for instance by adding a constant value greater to the absolute value of the minimum value of the strain reached throughout the map, or by taking the exponential of the strain distribution, deconvolving it and taking the logarithm of the result. Both strategies lead to the same conclusion. The result obtained with the second strategy is given in Figure 18. Figure 18-a is a closeup view of the cross-section 
of the strain maps in the same region as in Figure 17-b for different numbers of iterations, so the results of both the proposed procedure and the RL algorithm can be compared. A regular sharpening of the details is observed, but without convergence, even after 50 iterations. Some fake details also appear since some lobes progressively emerge from zones, which are very smooth in the initial map, see for instance on the left-hand side of Figure 18-a.

a- closeup view between $x_{1}=270$ pixels and $x_{1}=420$ pixels

for various iteration values (increment between two dashed lines: 10 iterations)
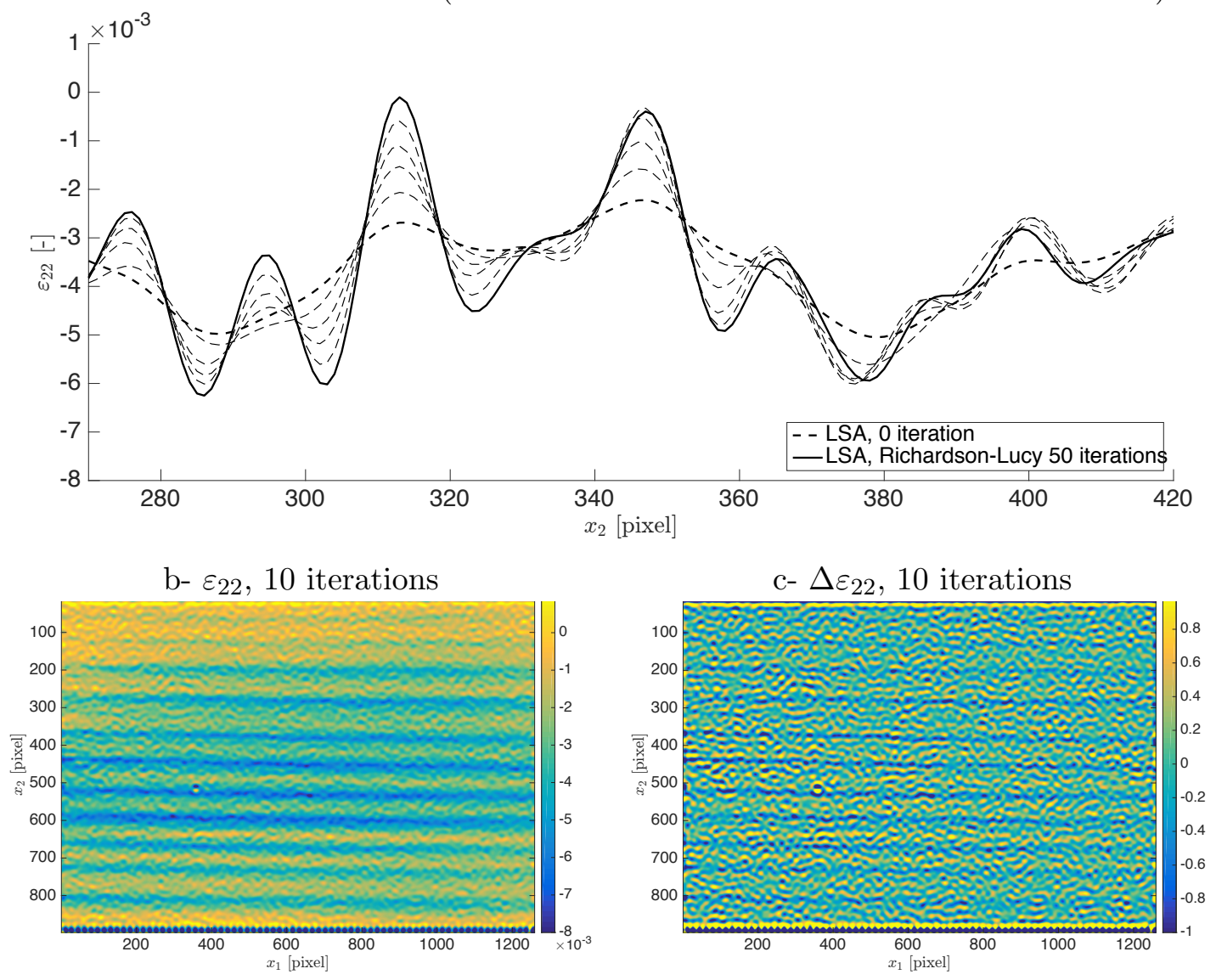

d- $\varepsilon_{22}, 50$ iterations
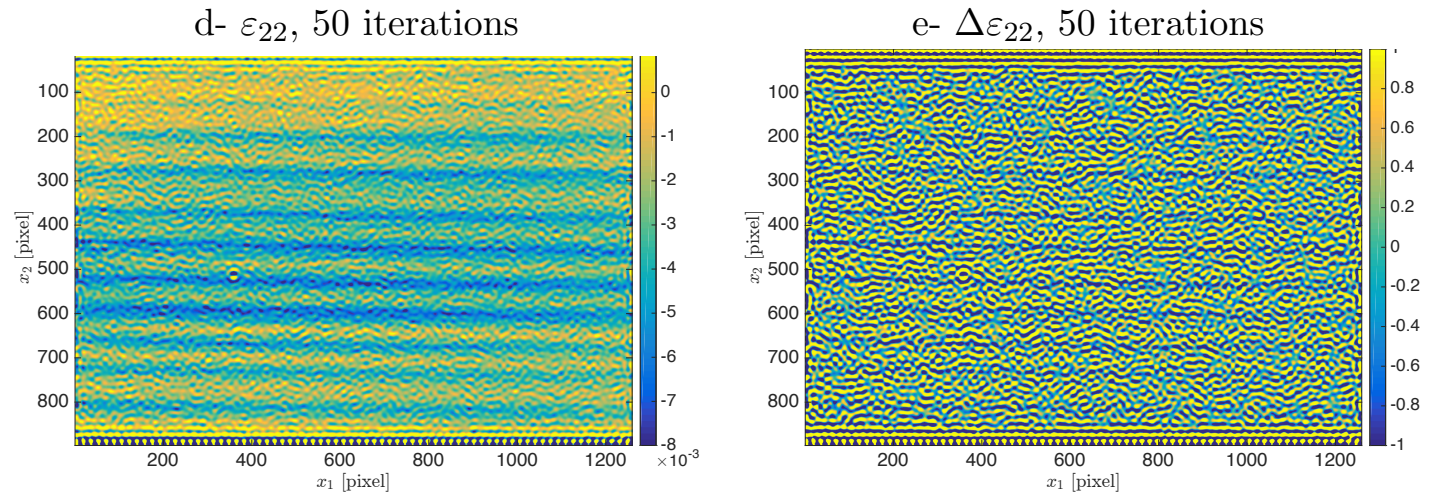

Fig. 18: $\varepsilon_{22}$ distribution during the RL deconvolution procedure, $x_{1}=500$ pixels, $\sigma=p$ 
Figures 18b to 18e show various deconvolved strain maps (on the left) along with the difference between deconvolved and initial strain maps (on the right). It can be seen that the details are enhanced after ten iterations, but the problem is to have a criterion in order to fix the maximum number of iterations, with which the final result can be given. Indeed, it can be seen in Figures 18-d-e that noise continues to increase between 10 to 50 iterations. The actual details are therefore progressively lost in the noise. This illustrates that the RL algorithm does not converge for noisy strain maps, as discussed in [23]. Hence apart from saying that the map is pleasant to the eye, there is no criterion that can be used to say that the result obtained by applying the RL algorithm with a certain number of iterations is acceptable or not. By contrast, the deconvolution procedure proposed in the present paper to deconvolve this strain map rapidly converges, as discussed in the preceding section, so the number of iterations can be bounded.

\section{Conclusion}

A simple algorithm aimed at deconvolving maps obtained by minimizing the optical residual in the real domain with DIC, and in the Fourier domain with LSA, is proposed in this paper. It can be considered as a robust-to-noise extension of Van Cittert deconvolution, which relies on the small strain assumption. The data to be deconvolved are the displacements with DIC and the phases with LSA. The type of kernel changes from one technique to another since a Savitzky-Golay filter is used for DIC and a Gaussian function for LSA. The procedure converges even in the presence of noise if the size of the subset for DIC, and the size of the window for LSA, remain lower than a certain value. This value is the pitch of the checkerboard with the latter technique. The procedure can also be applied below a threshold frequency in order to reduce the noise in the final result. This also increases the value of the maximum subset size beyond which the procedure diverges for DIC.

The proposed algorithm is iterative. However it converges with a reasonable number of iterations since we chose 10 iterations at most in all the examples discussed in the paper, and this number is observed to be conservative.

The main conclusion of this work is that deconvolution cancels out the systematic error caused by convolution. This positive effect is obtained below a cutoff frequency, which depends on the subset size in DIC and on the apparent size of the Gaussian function used in LSA. Another positive consequence is that the procedure diminishes the value of the spatial resolution (thus improves it). The drawback is that the noise level impairing the measurements increases, but not in the same proportion as the decrease of the spatial resolution. Hence the compromise between measurement resolution and spatial resolution is significantly improved, which makes this deconvolution procedure potentially useful in situations for which localized strain gradients occur. The main limitation of this deconvolution procedure is that it does not converge if too high values for the subset size in DIC, or for the standard deviation of the Gaussian function in LSA, are employed. It is also worth noting that deconvolution is applied for displacement maps obtained pixelwise, without any interpolation between remote points such as centers of subsets in classic DIC. This is a limitation for DIC in terms of computation time, not really for LSA which provides by essence measurements defined pixelwise. Numerical simulations carried out on various synthetic deformed speckle and checkerboard images illustrate the efficiency of the deconvolution algorithm proposed in this paper. The procedure 
is also applied on experimental data. Finally, it is worth noting that we presented here in parallel results obtained with DIC and LSA to show that the same deconvolution procedure could be applied for both techniques, but we did not compare these two techniques. This comparison will be presented in a separate document.

\section{References}

[1] J. Réthoré. A fully integrated noise robust strategy for the identification of constitutive laws from digital images. International Journal for Numerical Methods in Engineering, 84(6):631-660, 2010.

[2] H. W. Schreier and M. Sutton. Systematic errors in digital image correlation due to undermatched subset shape functions. Experimental Mechanics, 42(3):303-310, 2002.

[3] A. Savitzky and M.J.E. Golay. Smoothing and differentiation of data by simplified leastsquares procedures. Analytical Chemistry, 36(3):1627-1639, 1964.

[4] F. Sur and M. Grédiac. Towards deconvolution to enhance the grid method for inplane strain measurement. Inverse Problems and Imaging, 8(1):259-291, 2014. American Institute of Mathematical Sciences.

[5] M. Grédiac, B. Blaysat, and F. Sur. A critical comparison of some metrological parameters characterizing local digital image correlation and grid method. Experimental Mechanics, 57(3):871-903, 2017.

[6] M. Sutton, J.J. Orteu, and H. Schreier. Image Correlation for Shape, Motion and Deformation Measurements. Basic Concepts, Theory and Applications. Springer, 2009.

[7] B. Pan, Z. Lu, and H. Xie. Mean intensity gradient: an effective global parameter for quality assessment of the speckle patterns used in digital image correlation. Optics and Lasers in Engineering, 48(4):469-77, 2010.

[8] J. Neggers, B. Blaysat, J.P.M. Hoefnagels, and M.G.D. Geers. On image gradients in digital image correlation. International Journal for Numerical Methods in Engineering, 105(4):243-260, 2016.

[9] https://sem.org/dic-challenge.

[10] Y. Surrel and B. Zhao. Simultaneous u-v displacement field measurement with a phaseshifting grid method. In Stupnicki J Pryputniewicz RJ, editor, Proceedings of the SPIE, the International Society for Optical Engineering, volume 2342. SPIE, 1994.

[11] G.F. Bomarito, J.D. Hochhalter, T.J. Ruggles, and A.H. Cannon. Increasing accuracy and precision of digital image correlation through pattern optimization. Optics and Lasers in Engineering, 91:73 - 85, 2017.

[12] M. J. Hytch, E. Snoeck, and R. Kilaas. Quantitative measurement of displacement and strain fields from HREM micrographs. Ultramicroscopy, 74:131-146, 1998. 
[13] R. H. Zhu, H. M. Xie, X. L. Dai, J. Zhu, and A. Jin. Residual stress measurement in thin films using a slitting method with geometric phase analysis under a dual beam (fib/sem) system. Measurement Science and Technology, 25(9):095003, 2014.

[14] X. Dai, H. Xie, H. Wang, C. Li, Z. Liu, and L. Wu. The geometric phase analysis method based on the local high resolution discrete Fourier transform for deformation measurement. Measurement Science and Technology, 25(2):025402, 2014.

[15] X. Dai, H. Xie, and H. Wang. Geometric phase analysis based on the windowed Fourier transform for the deformation field measurement. Optics and Laser Technology, 58(6):119-127, 2014.

[16] M. Grédiac, F. Sur, and B. Blaysat. The grid method for in-plane displacement and strain measurement: a review and analysis. Strain, 52(3):205-243, 2016.

[17] Q. Kemao. Windowed Fourier transform for fringe pattern analysis. Applied Optics, 43(13):2695-2702, 2004.

[18] Q. Kemao, H. Wang, and W. Gao. Windowed fourier transform for fringe pattern analysis: theoretical analyses. Applied Optics, 47(29):5408-5419, 2010.

[19] M. Grédiac, B. Blaysat, and F. Sur. Extracting displacement and strain fields from checkerboard images with the localized spectrum analysis. 2018. Experimental Mechanics Accepted.

[20] F. Sur and M. Grédiac. Influence of the analysis window on the metrological performance of the grid method. Journal of Mathematical Imaging and Vision, 56(3):472-498, 2016.

[21] J. L. Starck, E. Pantin, and F. Murtagh. Deconvolution in astronomy: A review. Publications of the Astronomical Society of the Pacific, 114(800):1051-1069, 2002.

[22] R.C. Gonzalez and R.E. Woods. Digital Image Processing (3rd Edition). Prentice-Hall, 2006.

[23] M. Grédiac, F. Sur, C. Badulescu, and J.-D. Mathias. Using deconvolution to improve the metrological performance of the grid method. Optics and Lasers in Engineering, 51(6):716-734, 2013.

[24] F. Murtagh, E. Pantin, and J.-L. Starck. Deconvolution and blind deconvolution in astronomy. In P. Campisi and K. Egiazarian, editors, Blind Image Deconvolution: Theory and Applications, pages 277-316. Taylor and Francis, 2007.

[25] P. Sagaut. Structural modeling. In Large Eddy Simulation for Incompressible Flows: An Introduction, pages 183-240. Springer Berlin Heidelberg, 2002.

[26] F. Sur, B. Blaysat, and M. Grédiac. Rendering deformed speckle images with a Boolean model. Journal of Mathematical Imaging and Vision, 60(5):634-650, 2018.

[27] P. Reu. All about speckles: Aliasing. Experimental Techniques, 38(5):1-3, 2014. 
[28] M. Grédiac and F. Sur. Effect of sensor noise on the resolution and spatial resolution of the displacement and strain maps obtained with the grid method. Strain, 50(1):1-27, 2014. Paper invited for the 50th anniversary of the journal.

[29] R.B. Lehoucq, P.L. Reu, and D.Z. Turner. The effect of the ill-posed problem on quantitative error assessment in digital image correlation. Experimental Mechanics, 2017. Accepted, online.

[30] R.W. Schafer. What is a Savitzky-Golay filter? (lecture notes). IEEE Signal Processing Magazine, 28(4):111-117, 2011.

[31] E. W. Grafarend. Linear and Nonlinear Models: Fixed Effects, Random Effects, and Mixed Models. Walter de Gruyter, 2006.

[32] J.L. Piro and M. Grédiac. Producing and transferring low-spatial-frequency grids for measuring displacement fields with moiré and grid methods. Experimental Techniques, 28(4):23-26, 2004.

[33] F. Sur, B. Blaysat, and M. Grédiac. Determining displacement and strain maps immune from aliasing effect with the grid method. Optics and Lasers in Engineering, 86:317-328, 2016.

[34] F. Pierron and M. Grédiac. The virtual fields method. Springer, 2012. 517 pages, ISBN 978-1-4614-1823-8.

[35] W. H. Richardson. Bayesian-based iterative method of image restoration. Journal of the Optical Society of America, 62(1):55-59, 1972.

[36] L. B. Lucy. An iterative technique for the rectification of observed distributions. Astronomical Journal, 79(6):745-754, 1974.

[37] International vocabulary of metrology. Basic and general concepts and associated terms, 2008. Third edition.

[38] A. Chrysochoos and Y. Surrel. Chapter 1. Basics of metrology and introduction to techniques. In M. Grédiac and F. Hild, editors, Full-field measurements and identification in solid mechanics, pages 1-29. Wiley, 2012.

[39] M. Bornert, F. Brémand, P. Doumalin, J.-C. Dupré, M. Fazzini, M. Grédiac, F. Hild, S. Mistou, J. Molimard, J.-J. Orteu, L. Robert, Y. Surrel, P. Vacher, and B. Wattrisse. Assessment of digital image correlation measurement errors: methodology and results. Experimental Mechanics, 49(3):353-370, 2009.

[40] L. Wittevrongel, P. Lava, S. V. Lomov, and D. Debruyne. A self adaptive global digital image correlation algorithm. Experimental Mechanics, 55(2):361-378, 2015.

[41] J. Blaber, B. Adair, and A. Antoniou. Ncorr: Open-source 2d digital image correlation matlab software. Experimental Mechanics, 2015. doi: 10.1007/s11340-015-0009-1.

[42] C. Badulescu, M. Bornert, J.-C. Dupré, S. Equis, M. Grédiac, J. Molimard, P. Picart, R. Rotinat, and V. Valle. Demodulation of spatial carrier images: Performance analysis of several algorithms. Experimental Mechanics, 53(8):1357-1370, 2013. 
[43] Y. Q. Wang, M.Sutton, H. Bruck, and H. W. Schreier. Quantitative error assessment in pattern matching: effects of intensity pattern noise, interpolation, strain and image contrast on motion measurements. Strain, 45(2):160 -178, 2009.

[44] Y. Su, Q. Zhang, Z. Gao, X. Xu, and X. Wu. Fourier-based interpolation bias prediction in digital image correlation. Optics Express, 23(15):19242-19260, 2015.

[45] Y. Su, Q. Zhang, X. Xu, and Z. Gao. Quality assessment of speckle patterns for DIC by consideration of both systematic errors and random errors. Optics and Lasers in Engineering, 86:132-142, 2016.

[46] G.F. Bomarito, J.D. Hochhalter, and T.J. Ruggles. Development of optimal multiscale patterns for digital image correlation via local grayscale variation. Experimental Mechanics, 2017. accepted, online.

[47] Y. Surrel. Moiré and grid methods: a signal-processing approach. In Stupnicki J Pryputniewicz RJ, editor, Interferometry'94: photomechanics, volume 2342. SPIE, 1994.

[48] Y. Surrel. Photomechanics, Topics in Applied Physic, volume 77, chapter Fringe Analysis, pages 55-102. Springer, 2000. 


\section{Appendix 1: Localized Spectrum Analysis applied to checker- board images}

This section is a brief reminder on the Localized Spectrum Analysis applied to checkerboard images in order to retrieve displacement fields. Full detail can be found in [19]. The first step of LSA consists in calculating the Windowed Fourier Transform (WFT) of the image. This calculation is performed in a particular case since the frequency is set to the value of the nominal frequency of the quasi-periodic marking. This leads to the following expression for the WFT, which is defined, for any $\left(x_{1}, x_{2}\right) \in \mathbb{R}^{2}$ and $\theta \in[0,2 \pi]$, by:

$$
\Psi\left(x_{1}, x_{2}, \theta\right)=\iint_{\mathbb{R}^{2}} s\left(\eta_{1}, \eta_{2}\right) w\left(x_{1}-\eta_{1}, x_{2}-\eta_{2}\right) e^{-2 i \pi f\left(\eta_{1} \cos (\theta)+\eta_{2} \sin (\theta)\right)} \mathrm{d} \eta_{1} \mathrm{~d} \eta_{2},
$$

where $\mathrm{w}$ is a $2 \mathrm{D}$ window function. We use here a Gaussian window characterized by its standard deviation $\sigma$ :

$$
w\left(x_{1}, x_{2}\right)=\frac{1}{2 \pi \sigma^{2}} e^{\frac{-\left(x_{1}^{2}+x_{2}^{2}\right)}{2 \sigma^{2}}} .
$$

It has been shown in [20] that $\sigma$ should be greater or equal to the pitch $p$ of the quasiperiodic pattern to process correctly the images. In this study, we consider patterns which are optimal in terms of sensor propagation, namely checkerboards [11, 46]. A typical checkerboard is shown in Figure 19. For checkerboards, it is shown in [19] that $\Psi$ shall be calculated along the diagonals $x_{1}^{\prime \prime}, x_{2}^{\prime \prime}$ of the natural symmetry axes $x_{1}^{\prime}, x_{2}^{\prime}$ of the checkerboard (see the axes shown in Figure 19), thus for $\theta=\alpha+\frac{\pi}{4}$ and $\theta=\alpha+\frac{3 \pi}{4}$ in Equation 28. $x_{1}, x_{2}$ correspond to the natural coordinate system of the camera sensor. It is different from $x_{1}^{\prime}, x_{2}^{\prime}$ because images of regular patterns may be prone to aliasing problems if these two coordinate systems $\left(\left(x_{1}, x_{2}\right)\right.$ and $\left.\left(x_{1}^{\prime}, x_{2}^{\prime}\right)\right)$ are aligned [33]. The WFT being applied twice: once along direction $x_{1}^{\prime \prime}$ and once along direction $x_{2}^{\prime \prime}$, two complex numbers are available at each pixel of coordinates $\left(x_{1}, x_{2}\right): \Psi\left(x_{1}, x_{2}, \alpha+\frac{\pi}{4}\right)$ and $\Psi\left(x_{1}, x_{2}, \alpha+\frac{3 \pi}{4}\right)$. 


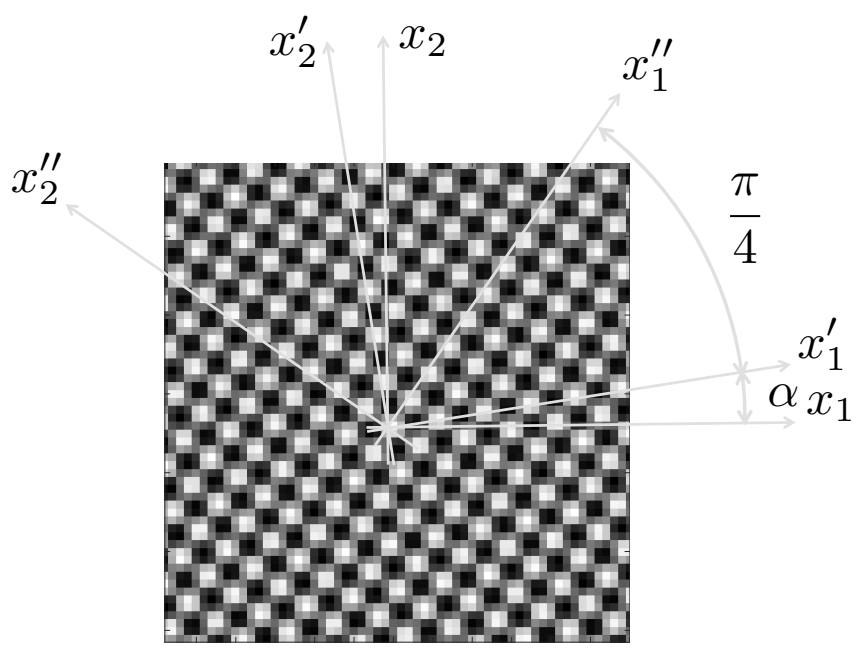

Fig. 19: Checkerboard and different coordinate systems.

The second step of the method consists in extracting and unwrapping the two phases of both the reference and the deformed images along the $x_{1}^{\prime \prime}$ - and $x_{2}^{\prime \prime}$-directions. These quantities are generally considered to be equal to the arguments of the WFT (here $\Psi\left(x_{1}, x_{2}, \alpha+\frac{\pi}{4}\right)$ and

$\left.\Psi\left(x_{1}, x_{2}, \alpha+\frac{3 \pi}{4}\right)\right),[47,48,16]$. They are then expressed in the $x_{1}, x_{2}$ coordinate system by a change of basis, and the sought displacements along the natural symmetry axes of the camera sensor $x_{1}, x_{2}$ are given by

$$
\underline{u}\left(x_{1}, x_{2}\right)=-\frac{p}{2 \pi}\left(\underline{\Phi}_{g}(\underline{x}+\underline{u})-\underline{\Phi}_{f}(\underline{x})\right) .
$$

$\underline{u}$ is the displacement at any point of coordinates $\underline{x} . \underline{\Phi}_{g}$ and $\underline{\Phi}_{f}$ are the phases of the periodic pattern of the current (or deformed) and reference images, respectively. The unknown displacement is involved in both parts of Equation 30, so it can be found by using a fixedpoint algorithm, which generally converges after one iteration only [16]. It has been recently demonstrated in [4] that this result is only an approximation. Indeed, regardless of noise, the arguments of the WFTs discussed above are equal to the sought phases convolved by the window $w$ used in the WFT.

\section{Appendix 2: definition of the metrological parameters used in this study}

Three metrological parameters are first discussed in this paper, namely the measurement resolution, the bias and the spatial resolution. A fourth one named metrological efficiency indicator is also introduced. It is defined by the product of the first and last quantities. All these parameters are throughly defined in [5]. Their definitions are recalled below:

Measurement resolution: in Ref. [37], the measurement resolution is defined by the smallest change in a quantity being measured that causes a perceptible change in the corresponding indication. More precisely, it is proposed in [38] to define it as the change in quantity being 
measured that causes a change in the corresponding indication greater than one standard deviation of the measurement noise, which enables us to quantify the measurement resolution. This definition is quite arbitrary, any other (reasonable) multiple of the standard deviation being also potentially acceptable, but the idea is that the resolution quantifies the smallest change not likely to be caused by measurement noise [38].

Bias: there are several causes for the systematic error observed with full-field measurement techniques. We consider here the so-called matching bias, which concerns both DIC and LSA. A classic way to assess it is to consider a synthetic reference sine function with a given amplitude, and to consider that the relative loss of amplitude quantifies this bias, as in Ref. $[39,40,41]$ for DIC or in $[42,23]$ for LSA. The bias is denoted by $\lambda$. The systematic error due to the interpolation function used to have both the reference and the deformed images in the same coordinate system $[43,44,45]$ is not considered here because it concerns only DIC and not LSA [16].

Spatial resolution: the spatial resolution denoted $\ell$ is defined here by the lowest period of a sinusoidal deformation that the technique is able to reproduce before losing a certain percentage of amplitude, in other words before the bias reaches a certain value, this quantity being chosen a priori [40]. The advantage of this definition is that it is not based on an arbitrary value for the subset size in DIC or for the window used while processing a periodic pattern with LSA. This makes it possible to compare the spatial resolution between these two techniques, whose principle is totally different. This definition of the spatial resolution holds here for the phase, and consequently for the displacement. It also holds for the phase derivatives and the strain components if no smoothing is performed before differentiating the phases and the displacements. Otherwise the spatial resolution is all the more impaired as the width of the filter increases.

Metrological efficiency indicator : For LSA, it has been proven that if the noise impairing the images is homoscedastic, the product between the displacement resolution and the spatial resolution is constant whatever the value of the size of the Gaussian window used to find the displacement $[16,5]$. This quantity is defined for a value of the bias $\lambda$ and denoted by $\alpha_{\lambda}$. It is observed that considering a more representative heteroscedastic noise makes $\alpha_{\lambda}$ nearly constant. Simulations also show that $\alpha_{\lambda}$ as defined here is nearly constant for DIC whatever the choice of the subset size. In conclusion, $\alpha_{\lambda}$ represents an indicator of the metrological performance of the measurement system, which is independent of the choice of the size of the window with LSA and of the subset with DIC. 